\section{SANDIA REPORT}

SAND87-0766 U UC-34

Unlimited Release

Printed July 1987
$73 \cdot 732.65975$

C. 1

\title{
A Numerical and Analytical Investigation of Rayleigh-Taylor Instability in a Solid Tungsten Plate
}

\author{
A. C. Robinson, J. W. Swegle
}

\section{Prepared by}

Sandia National Laboratories

Albuquerque, New Mexico 87185 and Livermore, California 94550

for the United States Department of Energy

under Contract DE-ACO4-76DPO0789 
Issued by Sandia National Laboratories, operated for the United States Department of Energy by Sandia Corporation.

NOTICE: This report was prepared as an account of work sponsored by an agency of the United States Government. Neither the United States Government nor any agency thereof, nor any of their employees, nor any of their ment nor any agency thereof, nor any of their employees, nor any of their
contractors, subcontractors, or their employees, makes any warranty, express contractors, subcontractors, or their employees, makes any warranty, express
or implied, or assumes any legal liability or responsibility for the accuracy, or implied, or assumes any legal liability or responsibility for the accuracy, completeness, or usefulness of any information, apparatus, product, or prorights. Reference herein to any specific commercial product, process, or service by trade name, trademark, manufacturer, or otherwise, does not necessarily constitute or imply its endorsement, recommendation, or favoring by the United States Government, any agency thereof or any of their by the United Government, any agency thereof or any of their contractors or subcontractors. The views and opinions expressed herein do not necessarily state or reflect those of the United States Government, any agency thereof or any of their contractors or subcontractors.

Printed in the United States of America Available from

National Technical Information Service

U.S. Department of Commerce

5285 Port Royal Road

Springfield, VA 22161

NTIS price codes

Printed copy: A04

Microfiche copy; A01 
Distribution

Category UC-34

SAND87-0766

Unlimited Distribution

Printed July 1987

\title{
A Numerical and Analytical Investigation of Rayleigh-Taylor Instability in a Solid Tungsten Plate
}

\author{
A. C. Robinson \\ Computational Physics \& Mechanics Division II \\ J. W. Swegle \\ Computational Physics \& Mechanics Division I \\ Sandia National Laboratories \\ Albuquerque, New Mexico 87185
}

\begin{abstract}
The Rayleigh-Taylor instability response of an elastic-plastic tungsten plate is investigated by numerical experiments and an approximate modal analysis. The so-called "minimum amplitude" instability criteria derived from plasticity analyses is shown to be incomplete as a general indicator of instability or stability at very large driving pressures. Model equations are derived which are able to reproduce the basic qualitative features of the observed instability response given by the numerical calculations.
\end{abstract}





\section{Contents}

$\begin{array}{ll}\text { 1. Introduction } & 1\end{array}$

2. Numerical Simulation of an Accelerated Tungsten Plate 3

3. Analytical Modeling 9

3.1 Model Equations . . . . . . . . . . . . . . . . . . . . . 9

3.2 Basis Functions . . . . . . . . . . . . . . . . . . . . 11

3.3 Application to a Newtonian Fluid and an Elastic Solid Layer . . . . . . . . . 12

3.4 Elastic-Plastic Analysis . . . . . . . . . . . . . . . . . . . . 14

3.5 Solutions to the Elastic-Plastic Model Equations . . . . . . . . . . . . 17

3.6 Rigid-Plastic Model with Linear Pressure Hardening . . . . . . . . . . . . . . 18

$\begin{array}{ll}\text { 4. Summary } & 19\end{array}$

$\begin{array}{ll}\text { References } & 49\end{array}$ 


\section{List of Figures}

1 Notation used in modal analysis of Rayleigh-Taylor stability of solid plates. . . . 20

2 Phase plane diagram for rigid-plastic stability equations. . . . . . . . . . 21

3 Notation and parameter space used in wavecode analysis. . . . . . . . . . 22

4 Sample wavecode output for an initial perturbation amplitude of $10 \mu \mathrm{m} . \ldots . .23$

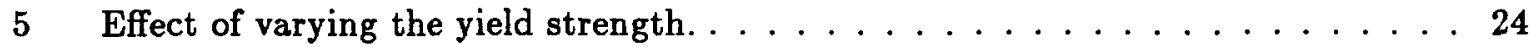

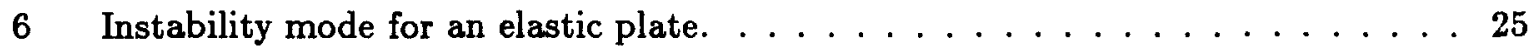

7 The effect of a pressure-dependent yield stress. . . . . . . . . . . 26

8 Variations in wavelength. . . . . . . . . . . . . . 27

9 Demonstration of wavelength of maximum growth. . . . . . . . . . 28

10 Amplitude-wavelength effect on stability. . . . . . . . . . . . . . 29

11 Response curves in unstable regime. . . . . . . . . . . . . . . . 30

12 Response curves in amplitude dependent stability regime. . . . . . . . . . 31

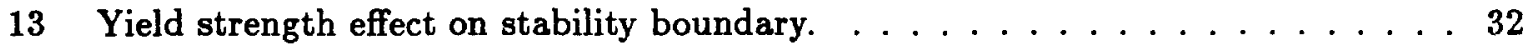

14 Driving pressure effect on stability boundary. . . . . . . . . . . 33

15 Instability growth vs. velocity. . . . . . . . . . . . . . . 34

16 Amplitude-wavelength effect on stability. . . . . . . . . . . 35

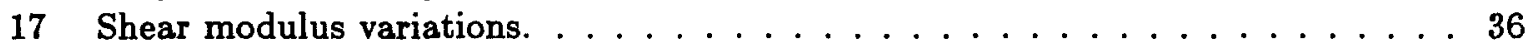

18 Wavelength variations in unstable regime. . . . . . . . . . . 37

19 Wavelength variations in stable regime. . . . . . . . . . . . . 38

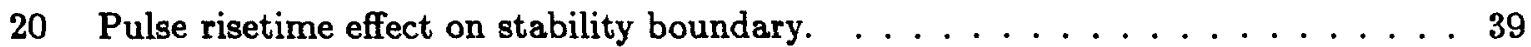

21 Newtonian fluid growth rates for $\rho_{2}=0 \ldots \ldots \ldots \ldots \ldots \ldots$

22 Elastic solid growth rates for $\rho_{2}=0 \ldots \ldots \ldots \ldots \ldots \ldots \ldots$

23 Comparison of limit stress versus wavenumber. . . . . . . . . . . . 42

24 Solution to model equations. $\left(\lambda / h=2.0 ; p_{m}=800 \mathrm{GPa}\right) \ldots \ldots \ldots$

25 Solution to model equations. $\left(\lambda / h=.5,1.0,1.5,2.0,2.5,3.0 ; \mathrm{P}_{m}=800 \mathrm{GPa}\right) \ldots 44$

26 Solution to model equations. $\left(\lambda / h=3,6,12,24,48 ; \mathrm{p}_{m}=800 \mathrm{GPa}\right) \ldots . .45$

27 A comparison of final growth rates in $(\mu s)^{-1} \ldots \ldots \ldots \ldots \ldots$

28 A comparison of plate velocity in $\mathrm{cm} / \mu \mathrm{s} . \ldots \ldots \ldots \ldots \ldots$

29 A comparison of relative velocity deviation $\delta v / v \equiv 2 \dot{q} / v \ldots \ldots \ldots \ldots$ 



\section{Introduction}

When a high density material is accelerated by a low density material in the direction of the positive density gradient, a classical Rayleigh-Taylor instability may develop along the interface between the two materials. The problem has been studied for some time in the case of fluids and the literature is voluminous (Sharp, 1984). We wish to study aspects of this instability behavior when the high density material is an elasticplastic solid pushed by a low density gas. We are interested principally in the regime where the driving pressure of the gas is much greater than the yield strength of the solid and wish to investigate how the properties of elasticity and plasticity for the solid plate may assist in delaying or suppressing the onset and growth of unstable modes.

Miles (1966) provided analytical estimates of the effect of solid properties on the growth of Rayleigh-Taylor instabilities. Using energy integral arguments and the inviscid fluid Rayleigh-Taylor eigenfunctions as basis functions for the velocity field, he determined two types of stability equations for two different regimes in the solid response of an incompressible elastic-plastic plate. The plate was assumed to be of thickness, $h$, have elastic shear modulus, $G$, and von-Mises yield stress in pure shear, $s_{1}$, related to the yield stress in unaxial tension, $Y$, by $Y=s_{1} \sqrt{3}$ (see Figure 1). A simple pressure boundary condition, $p_{0}$, was applied on the lower surface of the plate. He examined two limiting cases. The first case was for infinitesimal perturbations about an anisotropic state of stress in the plate. The steady state stress state for the solid was assumed to lie on the yield surface. For perturbation amplitudes much smaller than $h s_{1} / p_{0}$, Miles obtained, using the Prandtl-Reuss constitutive law, a perturbation amplitude equation of the form

$$
\ddot{q}-\left(g k-\frac{13}{4} \frac{G}{\rho} k^{2}\right) q=0
$$

where $q$ measures the amplitude of the unstable motion, the dot is a time derivative, $g$ is the body force acting on the plate, $k$ is the wavenumber of the spanwise perturbation and $\rho$ is the density. Since $p_{0}=\rho g h$, this implies that there exists a wavelength of maximum growth rate, $\lambda_{m}$, given by $\lambda_{m} / h=13 \pi G / p_{0}$. Thus, if the shear modulus is on the order of the driving pressure, the wavelength of maximum growth rate is roughly an order of magnitude larger than the thickness of the plate. A cutoff wavelength which is exactly half of the most unstable wavelength is also predicted. All wavelengths smaller than this cutoff wavelength would be predicted to be stable. For many metals this would imply that, unless the pressures driving the plate are on the order of $100 \mathrm{GPa}$ or more, the Rayleigh-Taylor response can be neglected as far as plate integrity is concerned provided initial perturbations are small. In order to provide an estimate for larger amplitudes, Miles assumed the von-Mises constitutive equations for plastic flow and using the same modal technique determined an equation of the form

$$
\ddot{q}-g k q=-4\left(1+e^{-k h}\right)^{-1}\left(k s_{1} / \rho\right) \operatorname{sgn} \dot{q}
$$


Letting $q=\delta h f(\tau)$, where $\tau=\sqrt{(g k)} t$ and $\delta=4\left(1+e^{-k h}\right)^{-1} s_{1} / p_{0}$, one obtains

$$
\ddot{f}(\tau)-f(\tau)=-\operatorname{sgn} \dot{f}(\tau)
$$

or, in integrated form,

$$
\dot{f}^{2}-(f-\operatorname{sgn} \dot{f})^{2}=\dot{f}_{0}^{2}-\left(f_{0}-\operatorname{sgn} \dot{f}_{0}\right)^{2}
$$

The level curves of Equation 4 are shown in Figure 2 along with the trajectory directions. For regions near the origin in the phase plane the motion is stable in the sense that the flow lines do not go to infinity for any values of the initial state. However, for large enough values of either $f(\tau)$ or $\dot{f}(\tau)$ the motion may follow an unstable trajectory. In particular, if one assumes $\dot{q}=0$ at the beginning of the motion one concludes that any initial $q>\delta h$ will lead to an unstable motion while any $q$ less than this cutoff value will be stable. Miles' analysis is the first known discussion of a minimum amplitude criterion for instability given an assumption of rigid-plastic material behavior. White (1973), in an informal report, discussed a unified analytical technique which included both the elastic and plastic response behavior seen in Miles' equations. Unfortunately, this work appears to have been largely unknown or ignored by subsequent authors. The relationship between White's theory and the distinct theory presented here in Section 3 will be discussed in a separate publication.

Barnes, et al. (1974) conducted an experimental study in which aluminum plates were accelerated by explosive gases expanding across a void. They found significant growth for perturbation wavelengths twice the thickness of the plate at a given initial amplitude. A single additional shot (Shot 2) at half the wavelength and half the amplitude resulted in significantly less growth. The result was ascribed to a wavelength effect with the growth of the shorter wavelength perturbation being damped much more by the yield strength of the solid just as in the case of viscosity in a fluid. Numerical calculations modeling these experiments indicated a significant dependence on yield strength.

Dienes (1978), using a fully nonlinear modal technique, determined an equation of motion for a rigid-plastic fluid and found that there was a minimum acceleration required for instability. His equations in the small amplitude regime are exactly the equations of Miles in the limit $h \rightarrow \infty$. Qualitatively the response was found to be the same for both linear and nonlinear modeling.

Drucker (1980) also obtained an equation similar to Equation 3 for $\dot{f}$ positive. His analysis results in a $\delta$ which does not depend on the wavelength but does depend on the shape of the perturbation. The analysis does not reduce to the fluid limit as the yield strength goes to zero. Drucker (1980) also produced an additional paper detailing various non-dimensional parameters of interest in the study of Rayleigh-Taylor instability in solids.

Due to the suggestion by Drucker that the initial amplitude rather than the wavelength was the dominant factor in the no growth result for Shot 2, Barnes, et al. (1980) conducted two additional shots. The first shot essentially verified the previous result 
of Shot 2 and the second shot had an initial perturbation wavelength 1.86 times that of Shot 2 but at the same small amplitude. In this case, little growth was observed. It was concluded that the minimum amplitude criterion emphasized by Drucker was apparently correct.

In this report, we first describe the results of a set of numerical experiments conducted using a Lagrangian finite-difference wavecode to compute the time-dependent motion of a solid tungsten plate loaded by a given pressure history on an initially perturbed surface. It will be seen that the theories discussed above are incomplete in the sense that all of the qualitative features seen in the numerical experiments cannot be explained by these theories. Subsequently, we introduce an approximate analytical technique which yields a coupled set of equations combining both elastic and plastic effects. We include in the derivation the effect of a small but non-zero density driving fluid for the sake of additional generality. A comparison is made between the results of the analysis using the approximate technique and the more precise modeling of the wavecode. It will be seen that the approximate analysis is able to reproduce the qualitative features of the solid plate response although the quantitative details differ.

\section{Numerical Simulation of an Accelerated Tungsten Plate}

This section describes an extensive parametric study of the stability characteristics of an accelerated tungsten plate using the Lagrangian finite-difference wavecode TOODY (Swegle, 1978). The study included variations in driving pressure profile, initial perturbation wavelength and amplitude, as well as yield strength and shear modulus variations. The conclusions to be reached by the study can be summarized as follows:

1. Increasing yield strength or decreasing driving pressure reduces instability growth rate and may stabilize the instability.

2. At a given time, there is a wavelength of maximum instability growth which increases as yield strength increases.

3. Stability curves dependent on yield strength separate stable and unstable regions in the perturbation amplitude-wavelength plane. Wavelengths larger than a cutoff wavelength, which increases as driving pressure decreases, are unstable at all amplitudes.

4. Simple scaling, such as dividing yield strength by driving pressure, does not hold.

5. Different behavior is seen depending on whether the driving pressure is constant or increasing. Thus, risetime of the driving pressure is a factor.

6. Low driving pressures can produce stability at realistic values of yield strength and perturbation amplitude. 
The study involves the calculated response of a section of the plate as shown in Figure 3. The driven surface of the plate has a sinusoidal perturbation of a given wavelength and amplitude imposed on it, while the opposite surface is flat. The plate has symmetry boundaries at the edges, so that a plate of infinite lateral extent is represented. The symmetry boundaries must fall at either a maximum or a minimum of the perturbation, so the minimum lateral extent of the calculation is one-half the wavelength of the perturbation. The driving gas is represented by an applied pressure boundary at the perturbed surface of the plate. The driving pressure varies in time, but is constant across the plate. This represents the extreme case of a driving gas of zero density and infinite sound speed. However, comparisons made with calculations in which a gas of representative density and sound speed was used to drive the plate showed no differences in the results. An alternate procedure would be to assume a flat plate and introduce perturbations by varying the driving pressure as a function of position on the surface. Non-uniformities in both the plate surface and the driving pressure will be present in the actual configuration.

The major part of the study consisted of a series of calculations in which the amplitude of the driving pressure, the amplitude and wavelength of the perturbation, and the yield strength of the plate were varied. Perturbation amplitudes ranged from 0.2 angstroms to $20 \mu$, wavelengths varied from $1 / 16$ of the plate thickness to 16 times the plate thickness, and the driving pressure varied from 80 to $1200 \mathrm{GPa}(0.8$ to $12 \mathrm{Mbar}$ ). In most cases the time variation of the pressure consisted of a linear ramp to the maximum pressure over 2.5 microseconds, after which the pressure remained constant. The plate material used in the study was tungsten. The nonlinear material model assumed a Mie-Gruneisen equation of state with a constant $\rho \Gamma$ product combined with linear shock velocity-particle velocity Hugoniot relation and a constant Poisson's ratio. The material properties are shown in Table I. The ambient yield strength of tungsten is 2.2 GPa, but material strength was treated as a parameter and varied from the ambient value to infinity. Since the aim of the present study was to elucidate the effect of the various parameters on the stability of the plate, a constant yield strength was assumed in each calculation, with the value varying between calculations. A more realistic yield model would be desirable for detailed calculations of an explicit configuration, but the elastic-perfectly plastic model is preferable for a parameter study, since the effect of the various parameters is most clearly seen if one parameter is varied while the others remain constant. A complex yield model results in difficulty in separating effects due to the time variation of the yield strength as opposed to variations in the other parameters.

Figure 4 shows mesh plots for a typical calculation. The parameter of interest is the overall variation in the flatness of the driven surface, which is initially equal to twice the amplitude of the applied sinusoidal perturbation. Results consist of comparing time histories of the surface variation for different values of the initial parameters. In unstable cases, a barely discernible initial perturbation eventually grows into a deformation comparable in size to the plate thickness. At this point, mesh deformation causes the time step to go to zero, stopping the calculation. The calculations could be 
continued for a short time if rezoning were employed, but this is not necessary since the exponential growth rate of the perturbation has been well established by this time. In reality, the perturbation would continue to grow and the plate would very shortly disintegrate.

TABLE I

Tungsten Material Parameters

\begin{tabular}{||l|c||}
\hline $\begin{array}{l}\text { Density } \\
\rho_{o}\left(\mathrm{gm} / \mathrm{cm}^{3}\right)\end{array}$ & 19.3 \\
\hline $\begin{array}{l}\text { Bulk Sound Speed } \\
C_{\text {o }}(\mathrm{km} / \mathrm{sec})\end{array}$ & $\mathbf{4 . 0 3}$ \\
\hline $\begin{array}{l}\text { Slope of } U_{s}-U_{p} \text { Relation } \\
\text { S }\end{array}$ & 1.237 \\
\hline $\begin{array}{l}\text { Poisson's Ratio } \\
\nu\end{array}$ & .283 \\
\hline $\begin{array}{l}\text { Grüneisen Ratio } \\
\Gamma\end{array}$ & 1.54 \\
\hline $\begin{array}{l}\text { Initial Yield Strength } \\
\text { Yo (GPa) }\end{array}$ & 2.28 \\
\hline
\end{tabular}

Figure 5 shows the effect of varying the yield strength. In this and the next several figures, the driving pressure consists of a linear ramp over $2.5 \mu \mathrm{s}$ up to $1200 \mathrm{GPa}$ with this pressure thereafter held constant. As can be seen from the figure, increasing the yield strength delays the growth of the instability. At shorter wavelengths, the higher yield strength calculations become stable, in the sense that the perturbation ceases to grow after the pressure becomes constant. However, at longer wavelengths, even very large yield strengths are unstable, and the amount of time that the instability growth is delayed is not large. In fact, even the purely elastic case is unstable, with growth rates not too different from those at lower strengths. However, as Figure 6 shows, the deformation mode is different in this case, since no plastic flow can occur, and the plate thickness stays constant. If the pressure were removed, the plate would revert 
to its original shape. This is, of course, unrealistic from a physical standpoint in an additional way since tensile stresses greatly exceeding the fracture strength of the plate are generated.

As mentioned, most calculations assume a constant yield strength in the plate. In order to determine if any additional stabilizing mechanism might occur with a more complex yield model, comparisons were made between constant and varying yield strength models. Figure 7 shows representative results for $\lambda=4 H$ and $A=2 \times 10^{-5} \mathrm{~cm}$. The figures compare calculations in which all other parameters are held constant except the yield model. A pressure-dependent model is compared with calculations in which the constant yield strength is equal to the upper and lower bounds of the varying yield strength. As can be seen from the figure, the pressure-dependent calculation is bounded by the others. No additional stabilizing or fundamentally different behavior results when this additional yield strength model complexity is introduced. Thus, the results of the present study provide bounds on the material response and show the trends in the instability behavior.

Figure 8 demonstrates the effect of varying the wavelength of the perturbation. At low yield strengths the shortest wavelengths grow most rapidly, but as the yield strength increases, the situation reverses, and the short wavelengths are more stable. If yield strength becomes large enough, shorter wavelengths become stable. In general, yield strength is more effective at stabilizing short wavelengths than long wavelengths. Figure 9 shows that, for a given yield strength and a fixed time, there exists a wavelength for which the surface variation is the largest. In Figure 9 at a yield strength of $10 \mathrm{GPa}$, the wavelength of largest growth is near the plate thickness, while for the elastic case the corresponding wavelength is about eight times the plate thickness. The growth rate, however, for the unstable modes appears to be asymptotically largest for the smallest wavelengths.

A conclusion of the present numerical study of an elastic-plastic plate is that no single criterion based on either amplitude or wavelength alone is sufficient to determine stability. Instead, a map of the results in the amplitude-wavelength plane shows a region of stability and a region of instability, demarcated by a well-defined boundary. An example of such a map is shown in Figure 10, where various combinations of amplitude and wavelength are plotted for the case of a driving pressure ramping up to $1200 \mathrm{GPa}$ over 2.5 microseconds, and a yield strength of $20 \mathrm{GPa}$. The stability boundary shows that the amplitude which remains stable decreases as the wavelength increases. As the wavelength increases beyond a cutoff wavelength which is about 2.25 times the plate thickness, all perturbations are unstable. Figure 11 shows the surface variation for the points at a wavelength 2.5 times the thickness. Even the smallest amplitude, 0.2 angstroms, is unstable. However, Figure 12 shows that when the wavelength is decreased to 1.5 times the sample thickness, initial amplitudes less than 0.02 microns are stable, in the sense that the surface variation switches from an exponentially growing mode to an oscillatory mode when the driving pressure becomes constant. When the matrix of calculations is repeated for various yield strengths, a family of stability curves is determined, as shown in Figure 13. Increasing the yield strength raises the stability 
curve at short wavelengths, but does not change the value of the cutoff wavelength. Note that Figure 13 only delineates the regions of stability and instability without providing information on growth rates. Combining the results of the figure with the previous conclusions about yield strength dependence of the most unstable wavelength, a picture can be obtained of the stability behavior as wavelength is varied while other parameters are held constant. Very long wavelengths are unstable, but the growth at a given time increases as wavelength decreases until the most unstable wavelength is reached, and then growth decreases as wavelength decreases. For non-zero values of the yield strength, continued decrease of the wavelength results in crossing the stability boundary, and the perturbation becomes stable. We emphasize that maximum growth at a given time is not the same as maximum growth rate. The growth rate appears to be largest for the smallest wavelengths given enough time for this rate to be achieved. (Study Figure 9 in particular).

The preceding results have all involved a $1200 \mathrm{GPa}$ driving stress. While they provide previously undiscovered trends in stability behavior, they do not paint a very promising picture for the integrity of a real plate being accelerated by such a large pressure. The only situations which can be expected to be stable involve amplitude and yield strengths which are probably far removed from those attainable in reality. Although it can be expected that the yield strength of tungsten will increase beyond its ambient value as pressure increases, one would have to be very optimistic to hope that it will increase to the megabar range, or that driving configurations can be flat and homogeneous on a scale of hundredths of microns. However, the situation improves if variations in the driving pressure are considered. Figure 14 shows the stability curves for a $10 \mathrm{GPa}$ yield strength for various maximum driving pressures. In all cases the pressure history is a linear ramp to the maximum pressure over 2.5 microseconds. As the driving pressure is reduced, the boundary curves not only lie at much higher initial amplitudes, but the cutoff wavelength also increases. These curves are approaching realistic values of amplitude and yield strength, and thus motivate the use of small driving pressures. Of course, other considerations may favor the use of as high a driving pressure as possible. Most important of these could be the desire to achieve a high plate velocity in a short acceleration distance. In fact, Figure 15 shows that higher pressures are better from the standpoint of instability growth versus plate velocity. Naturally, the higher the pressure, the more rapidly both the plate velocity and the surface variation increase. However, the increase in velocity is faster than the increase in instability growth, and higher velocities are obtained at a given level of surface variation. However, the plate is unstable, and the velocities which are obtained before the plate deformation becomes unacceptable are limited. In any event, a stable acceleration process in which any velocity can be obtained, although at the expense of acceleration length, seems preferable.

Figure 16 shows that if the driving pressure is reduced to $2 \mathrm{Mb}$, stable acceleration is possible for micron plate flatnesses and inhomogeneities even if the yield strength does not increases beyond the ambient value. The figure also shows that the minimum amplitude instability criterion based on the parameter $\mathrm{Y} / \mathrm{P}$ does not scale. The reason 
can be seen in Figure 17, where the shear modulus is varied. Here various constant values of the shear modulus are compared, and it is found that shear modulus affects the stability in much the same manner as yield strength. The standard calculations use a nonlinear model in which the elastic moduli are pressure dependent. However, the moduli do not increase as rapidly as the pressure, so that shear modulus divided by pressure decreases as pressure increases. Thus, the results of Figure 16 are obtained, and the simple scaling law does not hold.

It has been mentioned several times to this point that the classification of stability is made depending on the behavior of the plate after the pressure becomes constant. An observation which can be made of all the many calculations which comprise this study is that surface variation grows while pressure is increasing, regardless of the value of the parameters. Figure 18 shows the situation outside the stability boundary, where growth continues after the driving pressure is constant. Here the yield strength is small and the wavelengths are beyond the wavelength of maximum growth, so the longer wavelengths have grown less at a given time. Figure 19 shows the case inside the stability boundary where the amplitude of the surface variation oscillates after the driving pressure becomes constant. The feature to note is that the amplitude and period of the oscillation increases as the wavelength increases toward the stability boundary. Thus the shortest wavelengths convert more quickly to a stable pattern when the pressure becomes constant. The longer wavelengths continue to grow as the larger amplitude oscillation is established. Thus, it appears that as the stability boundary is reached from the inside, the amplitude of this oscillation increases without bound, resulting in the continued unstable growth of the perturbation. A very careful examination of the figure reveals that before the pressure becomes constant, the shortest wavelengths are growing most rapidly, even though these wavelengths are the most stable when the driving pressure is constant. This emphasizes the fact that the behavior is very different depending on whether the pressure is increasing or is constant. Thus, it might be expected that stability would be affected by the amount of time that the pressure increases before becoming constant. Figure 20 shows that this is in fact the case. Here the variation is in the driving pressure. In all cases there is a linear ramp up to the maximum pressure, but both the risetime and the maximum pressure are varied. It is found that regions of stability and instability exist, with instability occurring for risetimes both longer and shorter than the optimum value.

The study to this point has revealed that although the general stability problem is more complicated than has been previously reported, the procedure used has uncovered general trends which are useful in design considerations for acceleration of metallic plates. Furthermore, although no data exist for the configurations considered here, the general agreement of the code and modeling technique with the results of Barnes, et al. (1974) on aluminum plates accelerated at pressure up to $100 \mathrm{kbar}$ suggest that the numerical modeling will predict the correct general trends in expected plate response for a given configuration and acceleration profile. The following section outlines an analytical model for the Rayleigh-Taylor response of accelerated plates which confirms the trends for plate response as described above. 


\section{Analytical Modeling}

None of the approximate analyses discussed in the introduction are able to reproduce all of the qualitative behavior seen in the numerical experiments. However, there seem to be qualitative similarities between the small amplitude analysis of Miles and the cutoff wavenumber behavior observed at very small amplitudes. The damping effect of the yield strength is also observed in the growth of the unstable modes and is suggestive of the damping indicated by the modal equations derived from a rigid-plastic analysis. This analysis also predicts certain amplitude criteria which are similar to the amplitude results given by the numerics for certain regions of the parameter space. However, the shape of the wavelength-amplitude stability boundary and its dependence upon yield strength and maximum pressure are not at all indicated by any of the analyses. The results of Miles, taken as a whole, however, are indicative of the type of model equations which we wish to have. One concludes that elasticity and plasticity should be combined in some consistent manner to produce the qualitative features observed numerically. In a sense we wish to modify the phase diagram in Figure 2 to include amplitudewavelength effects due to the presence of elasticity in the constitutive response. The notation of Section 3 is basically the same as in Section 2 with the following changes: $\mathrm{A}$, and $\mathrm{H}$ and $\mathrm{P}$ of Section 2 are $q(0), h$, and $\mathrm{p}$, respectively of Section 3.

\subsection{Model Equations}

The thrust of the following analysis is to derive model equation which will capture the essence of the observed phenomena seen in the numerical experiments. In particular we will develop a coupled set of equations which, depending upon the location in phase space of the solution, have characteristics similar to both the small amplitude elastic response equations of Miles and the plasticity-based equations of Miles or Drucker. For completeness we shall include in the analysis the effect of a small density of the driving fluid.

Figure 1 illustrates our notation. We assume a plate of thickness $h$ and density $\rho_{1}$ resting on an infinitely deep fluid of density $\rho_{2}$. A uniform acceleration field $g$ acting in the direction from the plate to the fluid produces a body force $\mathbf{b}=-\rho \mathrm{gj}$. This corresponds to the situation where the plate is accelerated by a pressure $p_{0}=\rho_{1} g h$. At the free surface of the plate the pressure is zero. We assume both the fluid and the plate to be incompressible so that the velocity field of the motion must be solenoidal. Mass conservation implies

$$
\operatorname{div} \mathbf{v}=0
$$

The momentum equation is

$$
\rho \dot{\mathbf{v}}=\operatorname{div} \mathbf{T}+\mathbf{b}
$$

where $\mathbf{T}$ is the Cauchy stress tensor, $\mathbf{b}$ is the body force and $\dot{\mathbf{v}}$ is the acceleration. The 
motion is assumed to be periodic in $x$ of wavelength $\lambda$ and wavenumber $k=2 \pi / \lambda$ with an imposed zero stress boundary condition on the upper surface and a zero velocity boundary condition as $y \rightarrow-\infty$. We assume the Cauchy stress tensor to be of the form

$$
\mathbf{T}=-p \mathbf{I}+\mathbf{S}
$$

where $\mathbf{S}$ is the deviatoric part of the stress, i.e. $\mathbf{S} \cdot \mathbf{I}=\mathbf{0}$. For an incompressible Newtonian fluid we have

$$
\mathrm{S}=2 \mu \mathrm{D}
$$

where $\mathbf{D}$ is the symmetric part of the rate of strain tensor and $\mu$ is the viscosity. A von-Mises plastic or rigid-plastic constitutive law has the form

$$
\mathbf{S}=\sqrt{2} s_{1} \mathbf{D} / \sqrt{\mathbf{D} \cdot \mathbf{D}}
$$

where $s_{1}$ is the yield strength in pure shear and $s_{1}=Y / \sqrt{3}$ where $Y$ is the yield strength in uniaxial tension. For an elastic-plastic incompressible material we shall assume a Prandtl-Reuss constitutive law of the form

$$
\dot{\mathbf{S}}+\beta \mathbf{S}=2 G \mathbf{D}
$$

where $G$ is the elastic shear modulus and

$$
\begin{aligned}
\beta & =\frac{G \dot{W}}{s_{1}^{2}} \text { provided } J_{2}=2 s_{1}^{2} \text { and } \dot{W}>0 \\
& =0 \text { provided } J_{2}<2 s_{1}^{2} \text { or } \dot{W}<0
\end{aligned}
$$

where $\dot{W}=\mathbf{S} \cdot \mathbf{D}$ and $J_{2}=\mathbf{S} \cdot \mathbf{S}$. Of course, for an inviscid fluid we take $\mathbf{S}$ identically equal to zero.

We shall derive our basic equations from a weak form of Equation 6 and use a Galerkin formulation to obtain simple equations of motion. Let $w$ be an arbitrary vector field. A weak form of the momentum equation is

$$
\int_{\partial V^{t}} \mathbf{s}(\mathbf{n}) \cdot \mathbf{w} d A+\int_{V^{t}}(\mathbf{b}-\rho \dot{\mathbf{v}}) \cdot \mathbf{w} d V=\int_{V^{t}} \mathbf{T} \cdot \operatorname{grad} \mathbf{w} d V
$$

for all test functions $w$. The superscript $t$ indicates that the volume depends on time. The quantity $\mathbf{s}(\mathbf{n})$ is the imposed boundary stress on the volume $V^{t}$. If, in particular, we take $\mathbf{w}=\mathbf{v}$ then Equation 13 represents a power expended theorem. The Galerkin technique, as used in standard finite-element numerical analysis algorithms, employs numerous very simple basis or shape functions with time-dependent amplitudes for the velocity $\mathbf{v}$ and computes the equations for the amplitudes by requiring Equation 13 to be satisfied when $w$ is replaced by a specific set of test functions which may be equal to the set of basis functions. In this analysis, we use only one fairly complicated basis function for the velocity field which one assumes is close to the expected motion. The 
equation of motion is then obtained by evaluating Equation 13 when $\mathbf{w}$ is set equal to the basis function. The basis function which we shall employ is the eigenfunction from the Rayleigh-Taylor linear stability problem for the inviscid fluid case. This eigenfunction is known in closed form and allows the Galerkin integrals to be evaluated explicitly to within certain approximations. Using this form of the basis function in Equation 13 with $\mathbf{S}=\mathbf{0}$ will give an ordinary differential equation for the amplitude $q$ which has the same characteristic values as the eigenvalues corresponding to the inviscid fluid Rayleigh-Taylor eigenfunction. In the case of a non-perfect fluid we have an extra set of boundary condition to be satisfied. Since the inviscid fluid eigenfunction does not satisfy these boundary conditions this leads to an inaccurate approximation for those modes for which the shear stress contribution is important. However, the qualitative features of the response may still be preserved using the modal technique. This will be illustrated in the cases of an incompressible Newtonian viscous fluid and incompressible elastic solid where the linear stability problems can be completed in closed form.

\subsection{Basis Functions}

The eigenfunctions corresponding to Rayleigh-Taylor instability for the case of a finite layer fluid of density $\rho_{1}$ resting on an infinite depth fluid of density $\rho_{2}$ can be calculated using standard linear stability analysis. We define these as the basis functions for the finite layer and the infinite depth fluid (subscripts 1 and 2, respectively) as

$$
\begin{aligned}
& \mathbf{m}_{\mathbf{1}}=\left\{\begin{array}{l}
\left(e^{-k y}+\frac{\rho_{2}}{\rho_{1}} e^{-k h} \frac{\cosh k y}{\sinh k h}\right) \sin k x \\
\left.e^{-k y}-\frac{\rho_{2}}{\rho_{1}} e^{-k h \frac{\sinh k y}{\sinh k h}}\right) \cos k x
\end{array}\right. \\
& \mathbf{m}_{\mathbf{2}}=\left\{\begin{array}{l}
-e^{k y} \sin k x \\
e^{k y} \cos k x
\end{array}\right.
\end{aligned}
$$

The velocity in each region is then given by

$$
\begin{aligned}
& \mathbf{v}_{1}=\dot{q} \mathbf{m}_{1} \\
& \mathbf{v}_{\mathbf{2}}=\dot{q} \mathbf{m}_{\mathbf{2}}
\end{aligned}
$$

The symmetric part of the velocity gradient tensor is given by

$$
\mathbf{D}_{1}=\left(\operatorname{grad} \mathbf{v}_{1}+\operatorname{grad} \mathbf{v}_{1}^{T}\right) / 2=\operatorname{grad}_{1}=\dot{q} k \mathbf{M}_{1}
$$

where

$$
\mathbf{M}_{1}=\frac{1}{k} \operatorname{grad} \mathbf{m}_{1}
$$

Examination of Equations 8, 9, and 10 suggests that the deviatoric stress in the plate be represented in the form

$$
\mathbf{S}_{1}=s(t) \mathbf{M}_{1} \text {. }
$$


Thus the base or non-perturbed state of stress in the plate is assumed to be isotropic. We note that this is not an optimal choice for the expected base stress state of a solid plate. It is, however, an allowable stress state and is required for consistency in subsequent analysis. Also

$$
\mathbf{S}_{2}=\mathbf{0}
$$

since we assume that the driving fluid can produce no shear stresses.

We use as our test functions $\mathbf{w}=\mathbf{m}_{\mathbf{1}}$ in region 1 and $\mathbf{w}=\mathbf{m}_{\mathbf{2}}$ in region 2 . The volume $V^{t}$ includes a single period in $x$ with $\eta_{2}<y<h+\eta_{1}$ in region 1 and $-\infty<$ $y<\eta_{2}$ in region 2. Substitution into Equation 13 yields

$$
\int_{V_{1}^{t}}\left(\mathbf{b}_{1}-\rho_{1} \dot{\mathbf{v}_{1}}\right) \cdot \mathbf{m}_{1} d V+\int_{V_{2}^{t}}\left(\mathbf{b}_{2}-\rho_{2} \dot{\mathbf{v}_{2}}\right) \cdot \mathbf{m}_{2} d V=\int_{V_{1}^{t}} s \mathbf{M}_{1}^{2} d V
$$

These integrals are to be evaluated explicitly neglecting all terms of second order in the amplitude, i.e. terms $O\left(q^{2}\right), O(q s), O(q \eta)$ etc. If $\mathrm{j}$ is the unit vector in the $y$ direction, the first order kinematic boundary conditions for the interface $\eta_{2}$ is $\frac{\partial \eta_{2}}{\partial t}=\mathbf{v} \cdot \mathbf{j}$ on $\mathrm{y}=0$. Thus the boundary is given by $\eta_{2}=q \mathrm{~m}_{1} \cdot \mathrm{j}$ evaluated on $y=0$. Similarly $\eta_{1}=q \mathrm{~m}_{1} \cdot \mathrm{j}$ evaluated on $y=h$. Some algebra leads to the following equation,

$$
\ddot{q}-g k \frac{\rho_{1}-\rho_{2}}{\rho_{1}+\rho_{2} \operatorname{coth} k h} q=\frac{-2 s k}{\rho_{1}+\rho_{2} \operatorname{coth} k h}\left\{1+\alpha+\frac{\alpha^{2} e^{2 k h}}{1+\alpha}\right\}
$$

where $\alpha=\epsilon e^{-k h}$ with $\epsilon=\rho_{2} /\left(\rho_{1} 2 \sinh k h\right)$. We shall in Section 3.4 assume $\epsilon \ll 1$. As expected, when $s=0$ this equation yields the exact dispersion relation for inviscid fluid wave motion along the interface between an infinite fluid of density $\rho_{2}$ and a covering fluid layer of thickness $h$ and density $\rho_{1}$.

\subsection{Application to a Newtonian Fluid and an Elastic Solid Layer}

We can compare the above approximation technique to the exact solution when the plate is an incompressible Newtonian fluid or elastic solid layer of infinite extent. In the Newtonian fluid case, Equation 8 holds, and we have $s=2 \mu_{1} k \dot{q}$ and $k h \rightarrow \infty$. The resulting equation is

$$
\ddot{q}-g k \frac{\rho_{1}-\rho_{2}}{\rho_{1}+\rho_{2}} q=\frac{-4 \mu_{1} k^{2} \dot{q}}{\rho_{1}+\rho_{2}}
$$

Assuming a solution proportional to $e^{\text {nt }}$ we obtain the characteristic equation

$$
n^{2}-g k \frac{\rho_{1}-\rho_{2}}{\rho_{1}+\rho_{2}}=\frac{-4 \mu_{1} k^{2} n}{\rho_{1}+\rho_{2}}
$$

This may be compared with the characteristic equation for $n$ derived by taking the limit $\mu_{2} \rightarrow 0$ in the dispersion relation from the linear stability analysis given by Chandrasekar (1961). This yields 


$$
n^{2}-g k \frac{\rho_{1}-\rho_{2}}{\rho_{1}+\rho_{2}}=\frac{-4 \mu_{1} k^{2} n}{\rho_{1}+\rho_{2}}+\frac{4 k^{4} \rho_{1} \nu_{1}^{2}}{\rho_{1}+\rho_{2}}\left(\sqrt{1+n / k^{2} \nu_{1}}-1\right)
$$

where $\nu_{1}=\mu_{1} / \rho_{1}$. The last term on the right hand side of Equation 26 can be considered the contribution to the characteristic equation due to the need to satisfy a velocity and stress continuity boundary conditions at the interface. One expects our approximate solution to be correct in the long wavelength limit, but wrong in the short wavelength limit since in the latter case viscosity effects should be more important. Taking leading order terms in a small $k$ expansion in Equation 26 results in Equation 25, the same equation obtained by the modal technique. For completeness we also give the short wavelength (large $k$ ) limit of Equation 26 which can be computed by taking two terms in the expansion of the square root in inverse powers of $k^{2}$. This yields

$$
\left(1+\frac{1}{2} \frac{\rho_{1}}{\rho_{1}+\rho_{2}}\right) n^{2}-g k \frac{\rho_{1}-\rho_{2}}{\rho_{1}+\rho_{2}}=\frac{-2 \rho_{1} \nu_{1} k^{2} n}{\rho_{1}+\rho_{2}}
$$

The inviscid fluid eigenfunction modal technique for the Newtonian fluid problem was used by Miles and Dienes (1966) to obtain Equation 25 for $\rho_{2}=0$. They determined the maximum growth rate to be $0.40\left(g^{2} / \nu\right)^{1 / 3}$ for the approximate analysis and $0.46\left(g^{2} / \nu\right)^{1 / 3}$ for the exact analysis. Figure 21 illustrates the growth rate curves as a function of wavenumber for the exact solution and for each of the approximations discussed above in the case $\rho_{2}=0$. Both the magnitude of the growth rate and the wavenumber of maximum growth rate are displaced from the exact values for each of the approximations. The example indicates that the inviscid fluid eigenfunction approximation technique can perform quantitatively well in the long wavelength limit, but may provide qualitatively correct response for shorter wavelengths.

A similar analysis can be easily obtained for the case of an elastic upper layer. The dispersion relation in this case will be obtained by replacing $\mu$ in the Newtonian fluid analysis by $G / n$. This follows directly from Equation 10 with $\beta=0$. One obtains the exact relation

$$
n^{2}-g k \frac{\rho_{1}-\rho_{2}}{\rho_{1}+\rho_{2}}=\frac{-4 G k^{2}}{\rho_{1}+\rho_{2}}+\frac{4 k^{4}(G / n)^{2} / \rho_{1}}{\rho_{1}+\rho_{2}}\left(\sqrt{1+n^{2} \rho_{1} / k^{2} G}-1\right)
$$

The long wavelength limit of this equation and the modal technique both yield

$$
n^{2}=g k \frac{\rho_{1}-\rho_{2}}{\rho_{1}+\rho_{2}}-\frac{4 G k^{2}}{\rho_{1}+\rho_{2}}
$$

The short wavelength limit equation is given by

$$
\left(1+\frac{1}{2} \frac{\rho_{1}}{\rho_{1}+\rho_{2}}\right) n^{2}=g k \frac{\rho_{1}-\rho_{2}}{\rho_{1}+\rho_{2}}-\frac{2 \rho_{1} G k^{2}}{\rho_{1}+\rho_{2}}
$$

The growth rate curves for each of these approximations are shown in Figure 22 for the case $\rho_{2}=0$. The scaling on this plot emphasizes the weaknesses of the long 
wavelength approximation due to the fact that the cutoff wavelength is not correctly predicted. However the comparison does indicate that replacing the constant $G$ in the long wavelength analysis by one half its nominal value may provide the best agreement with a more accurate analysis in so far as the cutoff wavelength and wavelength of maximum growth rate are concerned. This would also increase the maximum growth rate by a factor of two.

\subsection{Elastic-Plastic Analysis}

In the case of the elastic-plastic constitutive equation one cannot solve for $s$ explicitly as in the previous section. However, in order to derive an equation for $s$, we can still proceed with the basic Galerkin approach by multiplying Equation 10 by $\mathbf{M}_{\mathbf{1}}$ and integrating over the volume, $V_{1}^{t}$ to obtain

$$
\int_{V_{1}^{t}} \dot{\mathbf{S}} \cdot \mathbf{M}_{1} d V+\int_{V_{1}^{t}} \beta \mathbf{S} \cdot \mathbf{M}_{1} d V=\int_{V_{1}^{t}} 2 G \mathbf{D} \cdot \mathbf{M}_{1} d V
$$

The second integral on the left hand side need not be taken over the full volume, but only over those regions in which $\beta$ is non-zero. Due to the simple form of our basis function we must change the Prandtl-Reuss law to read

$$
\begin{aligned}
\beta & =\frac{G \dot{W}}{s_{1}^{2}} \text { provided } J_{2} \geq 2 s_{1}^{2} \text { and } \dot{W}>0 \\
& =0 \quad \text { provided } J_{2}<2 s_{1}^{2} \text { or } \dot{W}<0
\end{aligned}
$$

Therefore the region of integration in the second integral is bounded by the solution to the equation

$$
J_{2}=s^{2} \mathbf{M}_{1}^{2}=2 s_{1}^{2}
$$

That is, if $\hat{y}(x)$ is the solution to

$$
\left(s_{1} / s\right)^{2}=(1+\alpha)^{2} e^{-2 k y}+\alpha^{2} e^{2 k y}+2(1+\alpha) \alpha \cos 2 k x
$$

and as before we neglect quadratic terms in the amplitude, then we must integrate over the region $0 \leq y \leq \min (\max (0, \hat{y}), h)=\bar{y}$. The equation for the evolution of $s$ is thus

$$
\dot{s}+H(s \dot{q}) G k \dot{q}\left(s / s_{1}\right)^{2} \frac{\int_{0}^{\lambda} \int_{0}^{\nabla} \mathbf{M}_{1}^{4} d y d x}{\int_{0}^{\lambda} \int_{0}^{h} \mathbf{M}_{1}^{2} d y d x}=2 G k \dot{q}
$$

where $H$ is the Heaviside function. It should be recognized that the second term in Equation 36 is formally $O\left(\dot{q} s^{2}\right)$. However, since $s_{1}^{2}$ appears in the denominator as well and we consider motions for which $s / s_{1}$ is bounded, we may include this term without loss of consistency. (We shall see that when $\mathrm{x}=0, s / s_{1} \lesssim 1.55$ for initial conditions of interest). This second term represents the only nonlinear aspect of the analysis and leads to the amplitude effects to be discussed in Section 3.5. In order 
to obtain a simpler formulation of Equation 36 we make the further assumption that $\epsilon=\rho_{2} /\left(2 \rho_{1} \sinh k h\right) \ll 1$ and derive the explicit formula

$$
\begin{aligned}
\hat{y} & =-\frac{1}{2 k} \log \left(\frac{\left(s_{1} / s\right)}{1+\alpha}\right)^{2}+O(\epsilon) \cos 2 k x+O\left(\epsilon^{2}\right) \\
& =\tilde{y}+O(\epsilon) \cos 2 k x+O\left(\epsilon^{2}\right)
\end{aligned}
$$

Neglecting terms of order $\epsilon^{2}$ (but not $O(\epsilon q)$ ) in Equations 23 and 36 yields the coupled set of equations

$$
\begin{gathered}
\ddot{q}-g k A\left(1-\frac{2 \alpha \rho_{1}}{\rho_{1}+\rho_{2}}\right) q=\frac{-2 s k}{\rho_{1}+\rho_{2}}(1-A \alpha) \\
\dot{s}+\mathrm{H}(s \dot{q}) G k \dot{q}\left(\frac{s}{s_{1}}\right)^{2}(1+\alpha)^{2} \frac{\left(1-e^{-4 k \bar{y}}\right)}{\left(1-e^{-2 k h}\right)}=2 G k \dot{q}
\end{gathered}
$$

where $A=\left(\rho_{1}-\rho_{2}\right) /\left(\rho_{1}+\rho_{2}\right)$ is the Atwood number. One might expect more $O(\epsilon)$ terms to appear in Equation 40. However, it turns out that the $O(\epsilon)$ terms in $\mathbf{M}_{1}^{2}$ and $\mathbf{M}_{1}^{4}$ have a $\cos (2 k x)$ dependence and thus integrate to zero. If we ignore a precise application of the min-max function when $\tilde{y}$ is close to zero, the $O(\epsilon)$ term arising from Equation 38 also integrates out since the zeroeth order terms in the integrand are independent of $x$. This approximation leads to a consistency error in Equation 40 only during a small time interval as $\tilde{y}$ passes from zero to a finite value. If $\rho_{2}=0$ ( $\epsilon=0$ ) the formula for $\tilde{y}$ is fully consistent. In any case one expects the overall errors of the approximate modal technique to dominant any error introduced by this slight derivational inconsistency. The $(1+\alpha)^{2}$ term is left in unreduced form in order to simplify the solution of the limit stress as explained below.

It is clear that $\dot{s}$ will equal zero when the second term on the left hand side and the term on the right hand side of Equation 40 balance. The value of $s$ at which this balance is achieved will be termed the limit stress, $s^{*}$, and can be computed by solving

$$
\left(s / s_{1}\right)^{2}(1+\alpha)^{2} \frac{\left(1-e^{-4 k \tilde{y}}\right)}{\left(1-e^{-2 k h}\right)}=2
$$

for $s$. One obtains

$$
\left(\frac{s^{*}}{s_{1}}\right)^{2}=\frac{\left(\gamma+\sqrt{\gamma^{2}+1}\right)}{(1+\alpha)^{2}}
$$

where $\gamma=1-e^{-2 k h}$. Another computation shows that the corresponding $\tilde{y}^{*}$ is less than $h$. Thus the variable $\tilde{y}$ will approach $\tilde{y}^{*}$ as $s$ approaches $s^{*}$.

We may now look at various limits of the above equations in the case $\rho_{2}=0$ in order to compare with the equations derived by Miles. Equations 39 and 40 may be written in matrix form as 


$$
\frac{d}{d t}\left(\begin{array}{c}
q \\
\dot{q} \\
s
\end{array}\right)=\left(\begin{array}{ccc}
0 & 1 & 0 \\
g k & 0 & -2 k / \rho_{1} \\
0 & 2 G k f\left(s / s_{1}, \gamma, s \dot{q}\right) & 0
\end{array}\right)\left(\begin{array}{c}
q \\
\dot{q} \\
s
\end{array}\right)
$$

where the function $f\left(s / s_{1}, \gamma, s \dot{q}\right)=1$ for $|s| \leq s_{1}$ or $s \dot{q}<0$ and $f\left(s / s_{1}, \gamma\right)=1-$ $\left(\left(s / s_{1}\right)^{2}-\left(s / s_{1}\right)^{-2}\right) / 2 \gamma$ for $s_{1} \leq|s| \leq s^{*}=s_{1} \sqrt{\gamma+\sqrt{\gamma^{2}+1}}$ with $s \dot{q}>0$. For $|s|<s_{1}$ the nonlinear term in the stress rate equation is absent $(f=1)$ and we obtain a coupled set of linear equations which can be solved explicitly for any set of initial conditions. The general solution of Equations 43 for $f=1$ and $g$ constant is

$$
\left(\begin{array}{c}
q \\
\dot{q} \\
s
\end{array}\right)=c_{1}\left(\begin{array}{c}
2 k / \rho_{1} \\
0 \\
g k
\end{array}\right)+c_{2}\left(\begin{array}{c}
1 \\
n \\
2 G k
\end{array}\right) e^{n t}+c_{3}\left(\begin{array}{c}
1 \\
-n \\
2 G k
\end{array}\right) e^{-n t}
$$

where $\mathrm{n}=\sqrt{g k-4 G k^{2} / \rho_{1}}$ and the $c_{i}$ are constants. For initial conditions, $\dot{q}(0)=0$, $s(0)=0$, the solution is

$$
\left(\begin{array}{c}
q \\
\dot{q} \\
s
\end{array}\right)=\frac{q(0)}{n^{2}}\left(\begin{array}{c}
g k \cosh (n t)-4 G k^{2} / \rho \\
g k n \sinh (n t) \\
2 G g k^{2}(\cosh (n t)-1)
\end{array}\right)
$$

It is clear that the dominant exponentially growing mode satisfies the equation

$$
\ddot{q}-\left(g k-\frac{4 G k^{2}}{\rho_{1}}\right) q=0
$$

which is exactly Equation 1 derived by Miles except for the coefficient 4 in the place of 13/4. For $s=s^{*}$, we have $f=\dot{s}=0$ and we obtain

$$
\ddot{q}-g k q=-\frac{2 k s^{*}}{\rho_{1}}
$$

which may be compared with Equation 3 derived by Miles. The values of $s^{*} / s_{1}$ for Miles' solution and for the current analysis are shown in Figure 23. Respectively, the asymptotic limits as $k h \rightarrow \infty$ are 2 and $\sqrt{1+\sqrt{2}} \simeq 1.55$. The model equations derived in this section clearly retain essential characteristics of the response predicted by both of the analyses given by Miles and have thus achieved, at least partially, the desired goals set forth in the introduction to Section 3. The following section delineates various characteristics of the solutions of the model equations obtained by numerical integration. 


\subsection{Solutions to the Elastic-Plastic Model Equations}

Equations 43 derived in the previous section may be solved by numerical integration for a given set of initial conditions. The results presented in the following sections will be restricted to the particular initial conditions in which $q(0)$ is given, $\dot{q}(0)=0$ and $s(0)=0$. We shall describe solutions which correspond roughly to some of the results discussed in Section 2.

The parameters entering into the analysis were chosen as follows: $\rho_{1}=19.2 \mathrm{gm} / \mathrm{cc}$, $G=77.5 \mathrm{GPa}$ (approximately $1 / 2$ the nominal value), $h=0.2 \mathrm{~cm}$ and $\mathrm{Y}=10 \mathrm{GPa}$. The initial amplitude, wavelength and yield strength of the perturbation were varied as described below. The imposed pressure profile in all cases is a $2.5 \mu \mathrm{s}$ linear ramp to a maximum pressure, $\mathrm{p}_{m}$. Figure 24 shows, for an $800 \mathrm{GPa}$ maximum pressure, the numerically computed solution for the amplitude, $q$, velocity, $\dot{q}$ and stress variable, $s$ for a calculation sequence with a varying set of initial amplitudes for a perturbation wavelength of $\lambda / h=2.0$. One notes that for the largest amplitudes the perturbation grows in an unbounded way indicating strongly unstable behavior. For smaller amplitudes the perturbation grows slowly during the initial increasing phase of the pressure, but when the pressure stops increasing the perturbation amplitude oscillates. It is interesting to note that as long as $s$ stays somewhat below the limit value $s^{*}$, shown by the horizontal dotted line, the perturbation growth is inhibited. However once this saturation value of $s$ is obtained, exponential growth appears to quickly dominate. The dotted curves represent negative values of $\dot{q}$. Figure 25 shows for an initial amplitude of $1 \mu \mathrm{m}$ the results when the wavelength is varied from $0.5 h$ to $3 h$ in steps of $0.5 h$. The smaller wavelengths appear to be more stable. We see that for the oscillatory configurations the amplitude oscillates with a larger amplitude and longer period as the wavelength increases. The numerical simulations also exhibit this property as seen in Figure 19. Figure 26 shows the results as the wavelength is increased even further to $\lambda / h=3,6,12,24$ and 48. The case $\lambda / h=3$ is denoted by a square. Decreased growth corresponds to increasing $\lambda$ for the remaining curves. There is a slight dependence on wavelength of the limit stress at the longer wavelengths. Note, also, the existence at a given time of a wavelength of maximum growth. For the exponentially growing modes, the shortest wavelength unstable mode has, asymptotically, the largest growth rate just as discussed in Section 2. (Compare Figures 9 and 18.)

Figure 27 shows values of the growth rate, $\dot{q} / q$, in the amplitude-wavelength plane for those modes determined to be unstable at the time of $6 \mu \mathrm{s}$ or at the integration step achieving $q / h>1$, whichever came first. Instability or stability was determined according to whether the value $s=s^{*}$ was achieved or not, respectively. The four figures correspond to maximum pressures of $1200,800,500$ and $200 \mathrm{GPa}$. There is a striking qualitative similarity between these figures and Figure 14. However, note the significant quantitative difference in the minimum amplitude curves at the higher pressures and smaller wavelengths.

Figures 28 shows for the same configurations and times the plate velocity, $v$, obtained as the integral of the acceleration, $g$. Figure 29 shows a measure of the relative 
axial velocity spread in the plate, $\delta v / v$, assumed to be equal to $2 \dot{q} / v$. For plates which are unstable it seems clear that very large axial velocity spreads in the plate fragments are possible.

\subsection{Rigid-Plastic Model with Linear Pressure Hardening}

As an extended case of the rigid-plastic model equations represented by Equation 2, it is instructive to determine the modal stability equation for the case of a yield strength which depends upon pressure. We assume that the yield strength $Y$ varies linearly across the plate with the pressure. Thus for constant $d Y / d p$ we obtain

$$
Y=Y_{h}+(d Y / d p) p_{0}(h-y) / h
$$

where $Y_{h}$ is the nominal yield strength of the unloaded material on the back surface of the plate. The analysis follows in a straightforward way from Equations 9 and 13 using the same approximations as in Section 3.2. The important detail is that $s$ is now dependent upon $y$ and the corresponding integration over the product of the stress term and the test function must take this into account. One obtains

$$
\ddot{q}-g k q=-\frac{4 k \operatorname{sgn} \dot{q}}{\sqrt{3} \rho\left(1+e^{-k h}\right)}\left\{Y_{h}+(d Y / d p) p_{0}\left(\frac{1}{1-e^{-k h}}-\frac{1}{k h}\right)\right\}
$$

This is exactly Equation 2 except for an additional dependence of the right hand side on $k h$. It is easy to show that the following inequalities hold for all $(d Y / d p) p_{0} \geq 0$ and $k h \geq 0$ :

$$
Y_{h} \leq Y_{h}+(d Y / d p) p_{0}\left\{\frac{1}{1-e^{-k h}}-\frac{1}{k h}\right\} \leq Y_{h}+(d Y / d p) p_{0}
$$

The term in braces takes on a value of .5 at $k h=0$ and approaches 1.0 monotonically as $k h$ increases. Thus one finds, as expected, that the stabilizing effect of the linearly varying yield strength is bounded by that seen if the whole plate were composed of a material characterized by the least and the maximum yield strength of the linearly varying plate. This is consistent with the numerical calculations as discussed in Section 2. (See Figure 7). 


\section{Summary}

The numerical experiments and the analytical approximation discussed in Section 2 and 3 above are in substantial qualitative agreement. The basic point of agreement is that both elasticity and plasticity may play an important role in determining the location of the stability-instability boundary in an initial amplitude-wavelength stability diagram. The major discrepancy is that the approximate modal technique tends to greatly overestimate the critical amplitude relative to the numerical simulation. We suspect that this significant quantitative error is due to the assumption of an isotropic state of stress as a base state in the model equations and the greatly oversimplified constitutive equation for elastic-plastic flow as given by Equation 32. One way to empirically correct for this discrepancy in the simple modeling may be to choose an initial condition $s(0)=\delta s^{*}$ with $0<\delta<1$. However, this possibility has yet to be investigated.

In many practical cases $G / p_{0}$ is a large number and thus imposed perturbations which have a length scale on the order of the plate thickness will not grow unless a certain amplitude criterion is satisfied. Thus in this case an initial perturbation amplitude stability criterion can be a useful indicator of stability. However, if the pressure is large enough, the stabilizing influence of the elastic shear strength will be lost for wavelengths on the order of the plate thickness. The only effect of the yield strength will then be to retard the growth of perturbations, and thus in this sense, one would not expect to be able to drive a coherent plate no matter how small the initial perturbation. 


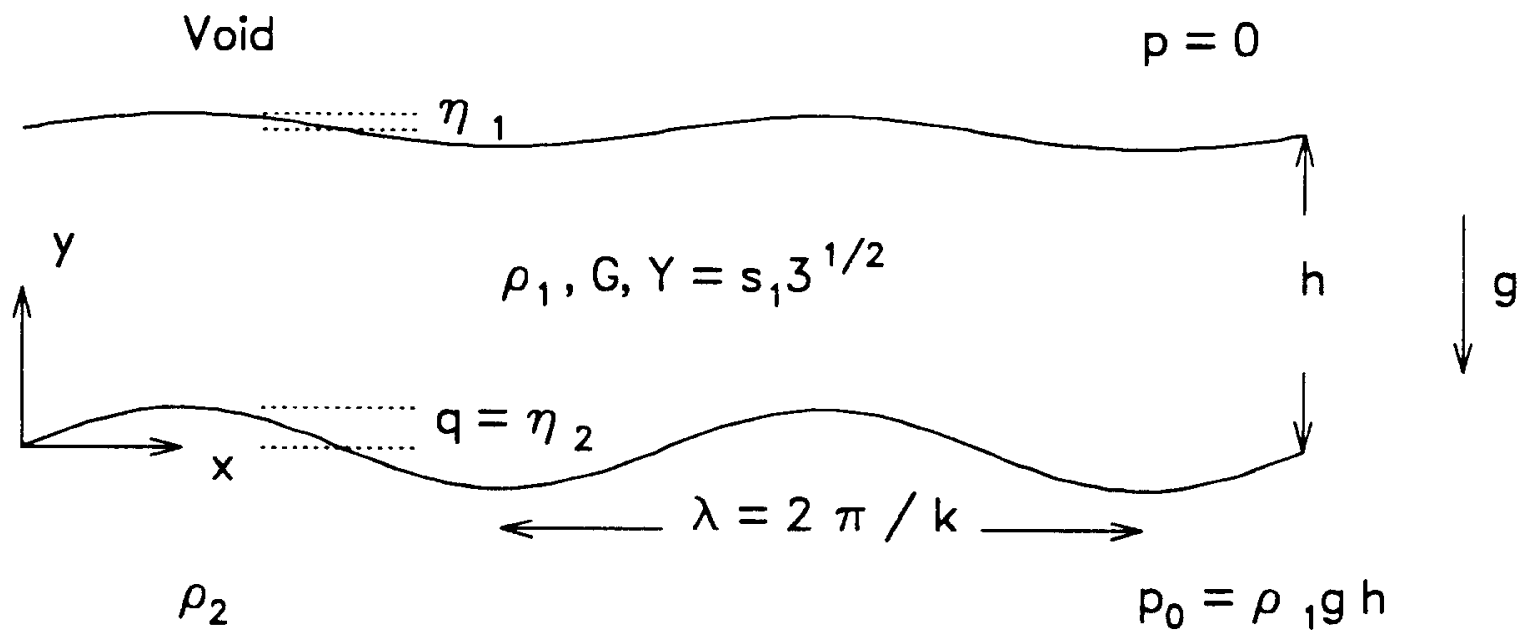

Figure 1: Notation used in modal analysis of Rayleigh-Taylor stability of solid plates. 


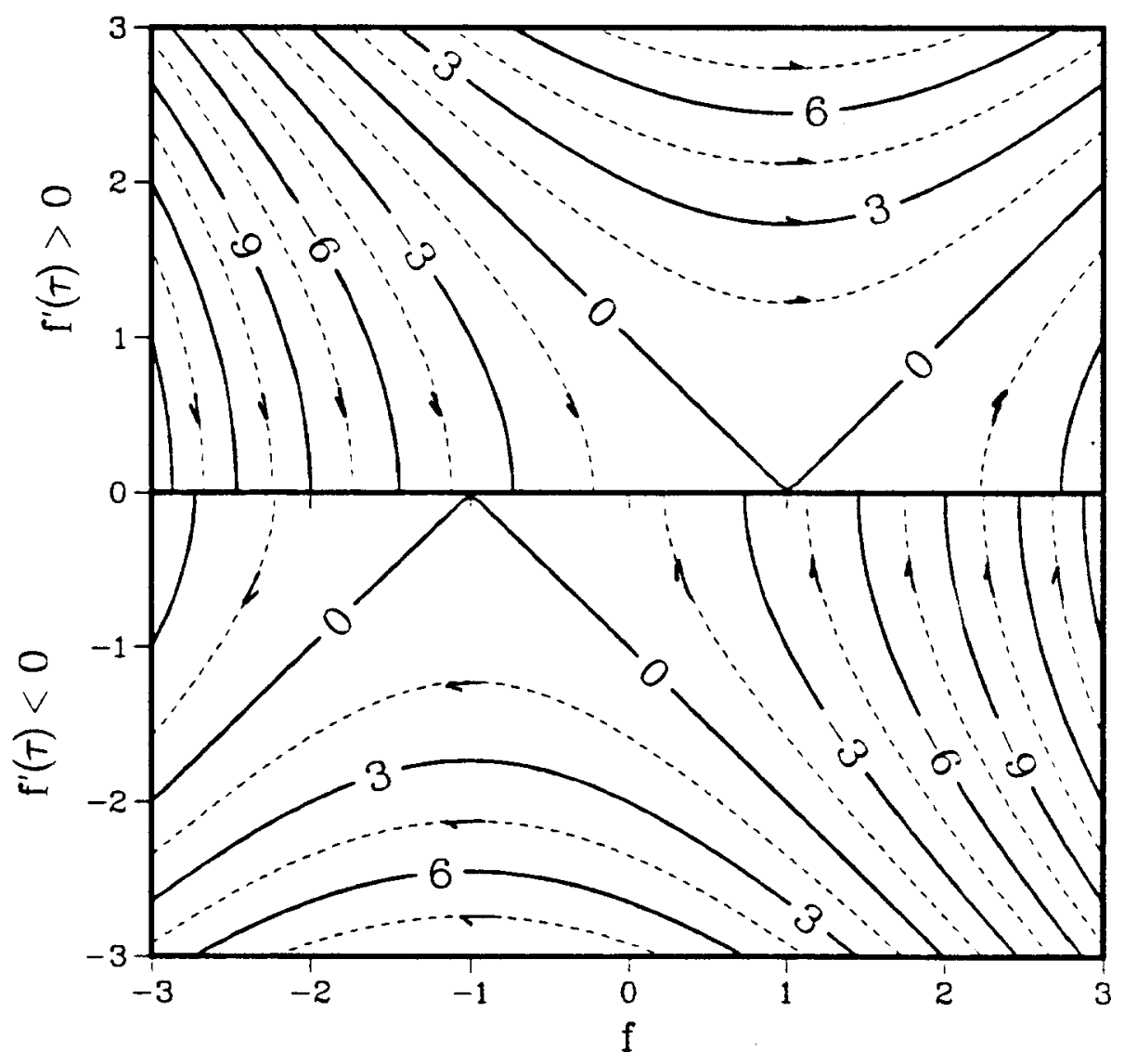

Figure 2: Phase plane diagram for rigid-plastic stability equations. Contour lines are level curves of $\dot{f}^{2}-(f-\operatorname{sgn} f)^{2}$. 


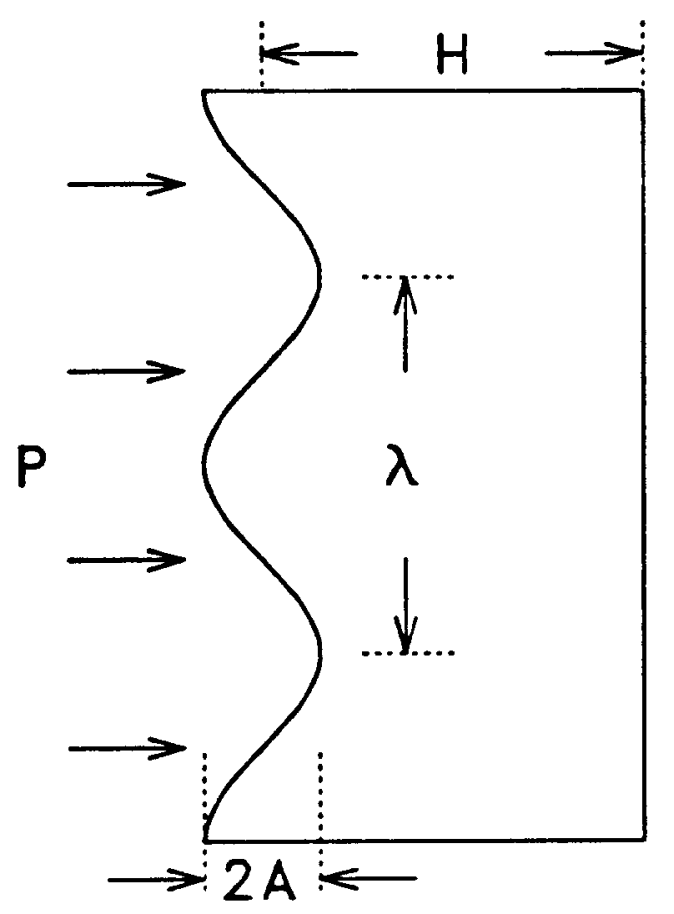

Parameter Variations
$A(\mu \mathrm{m}) \quad 2 \mathrm{E}-5-20$
$\lambda \quad H / 16-16 H$
$Y_{0}(G P a) \quad 2-\infty$
$P(G P a) \quad 80-1200$

Figure 3: Notation and parameter space used in wavecode analysis. 


$$
Y=10 \mathrm{GPa}
$$

$$
\mathrm{H}=2 \mathrm{~mm} \quad \mathrm{~A}=10 \mu \mathrm{m}
$$
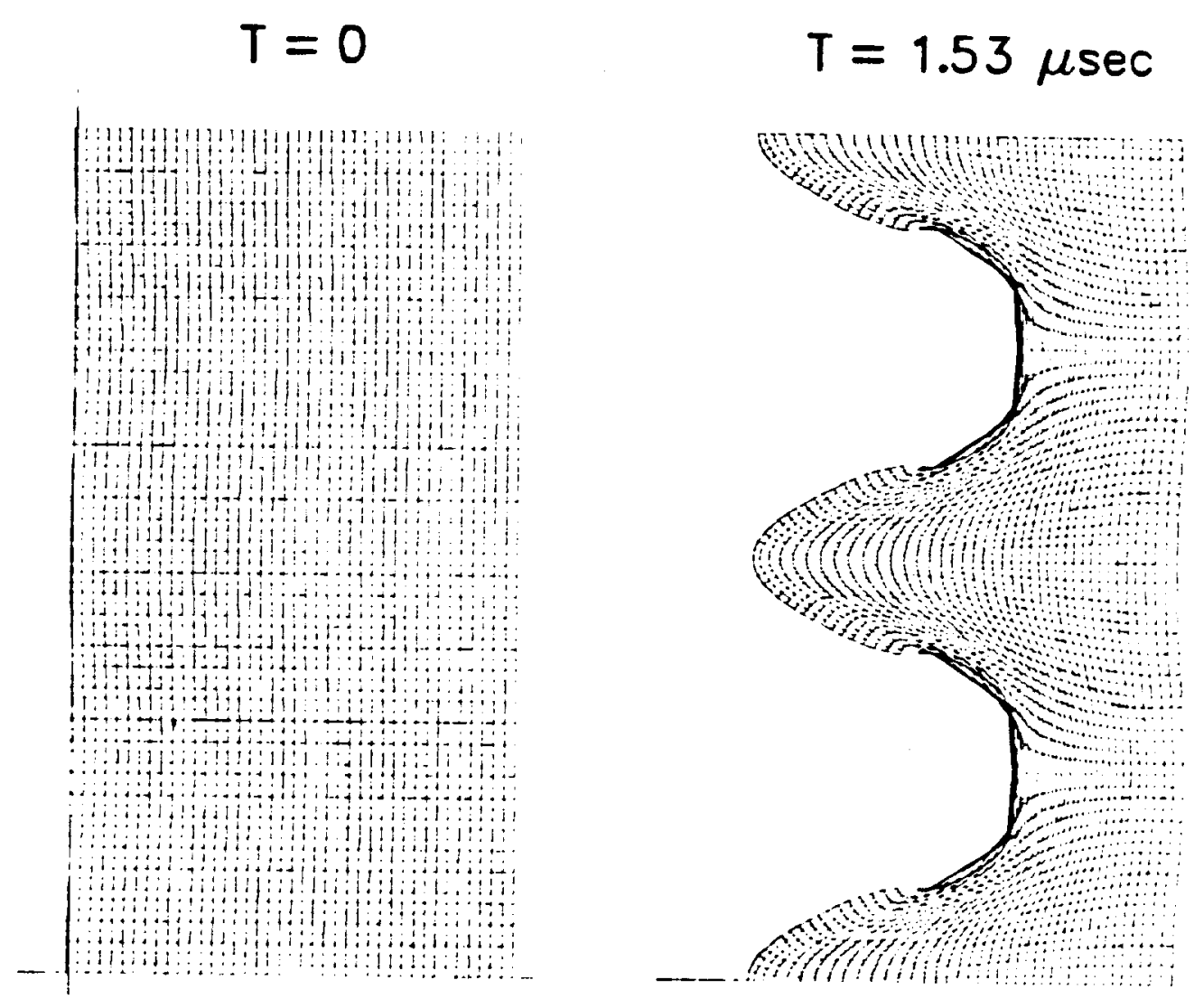

Figure 4: Sample wavecode output for an initial perturbation amplitude of $10 \mu \mathrm{m}$. 

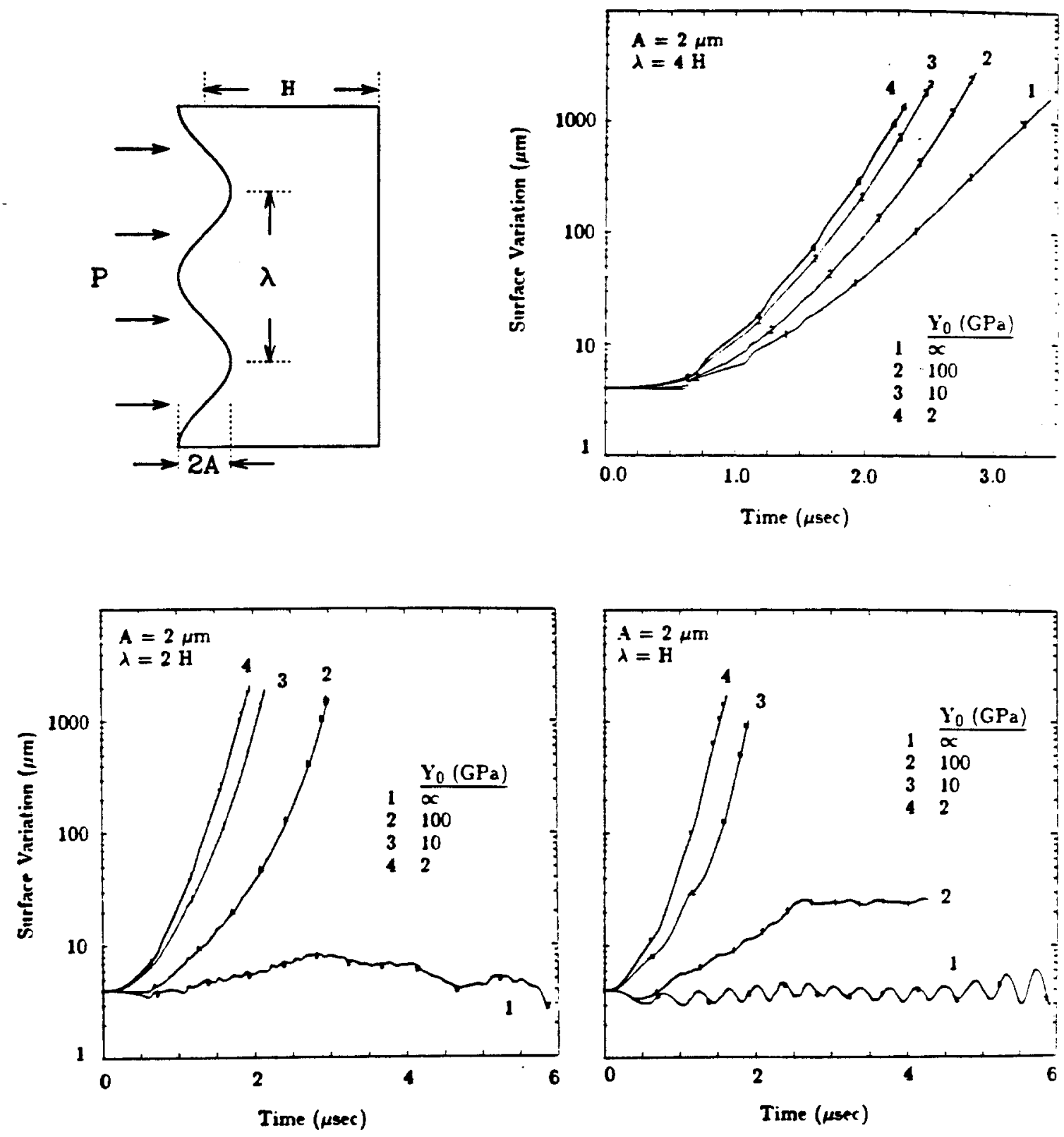

Figure 5: Effect of varying the yield strength. 


\section{$\lambda=4 \mathrm{H} \quad$ ELASTIC}

$\mathrm{H}=2 \mathrm{~mm} \quad \mathrm{~A}=.2 \mu \mathrm{m}$

$$
\mathrm{T}=4.33 \mu \mathrm{sec}
$$

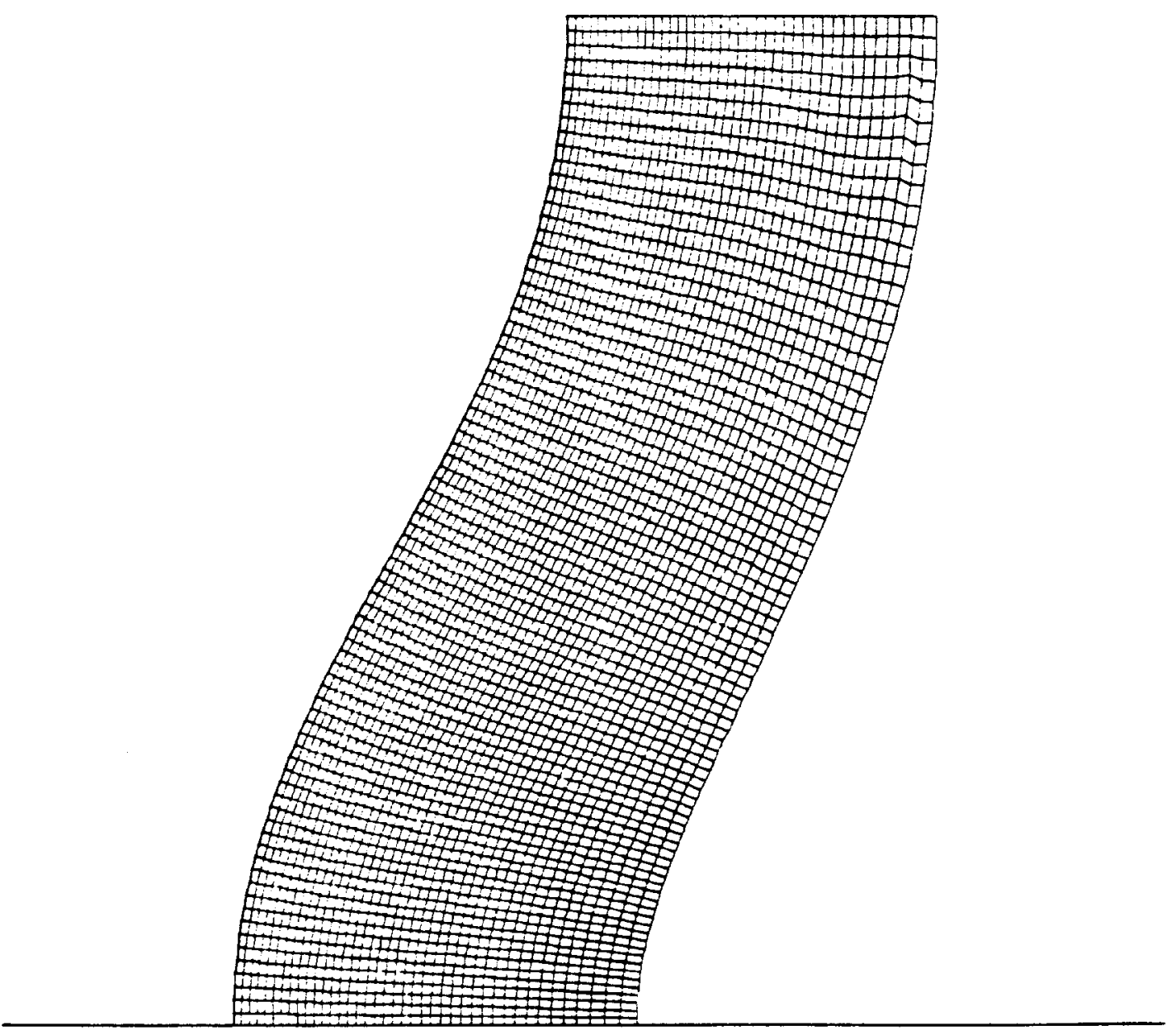

Figure 6: Instability mode for an elastic plate. 

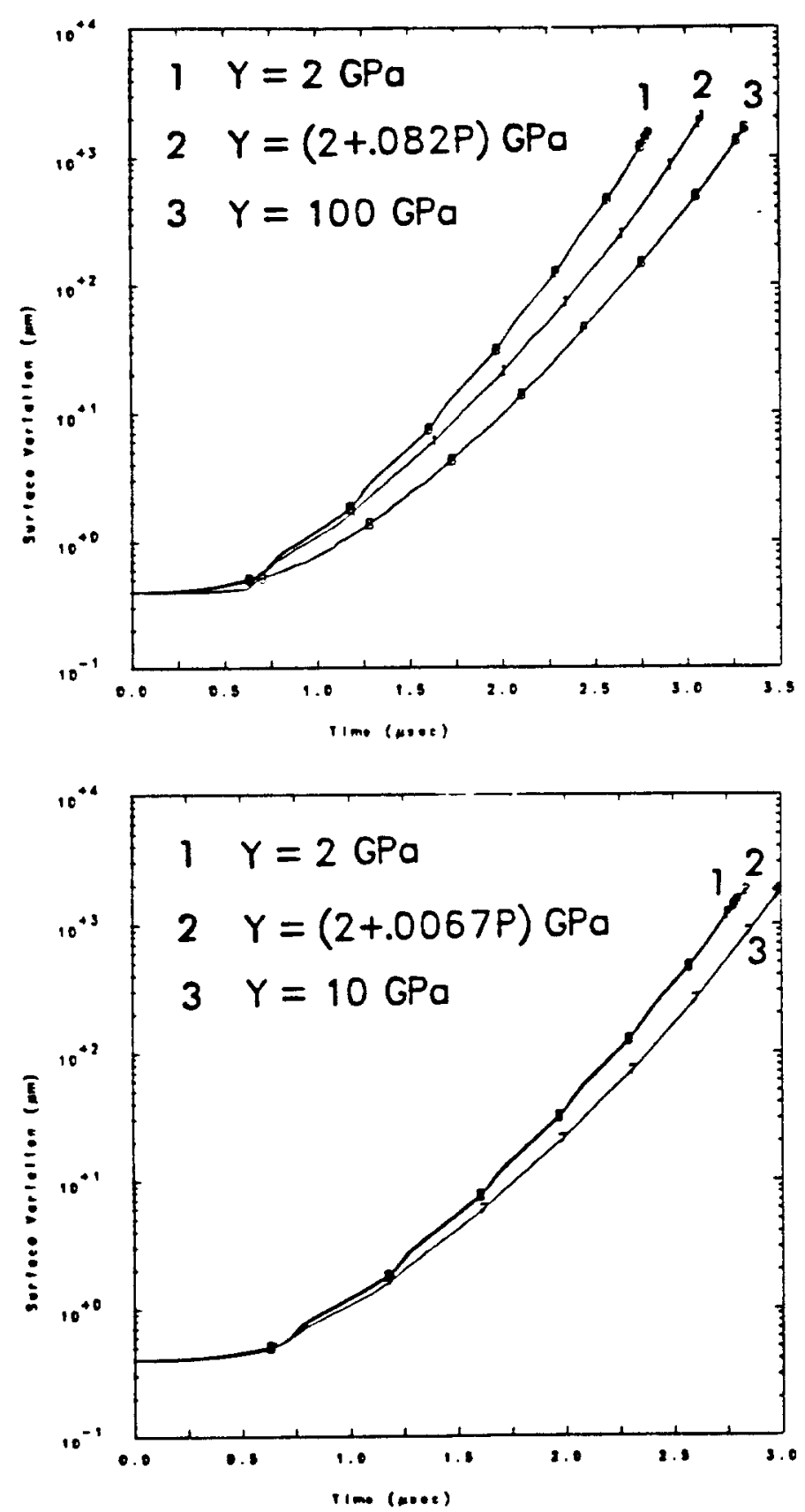

Figure 7: The effect of a pressure-dependent yield stress. 

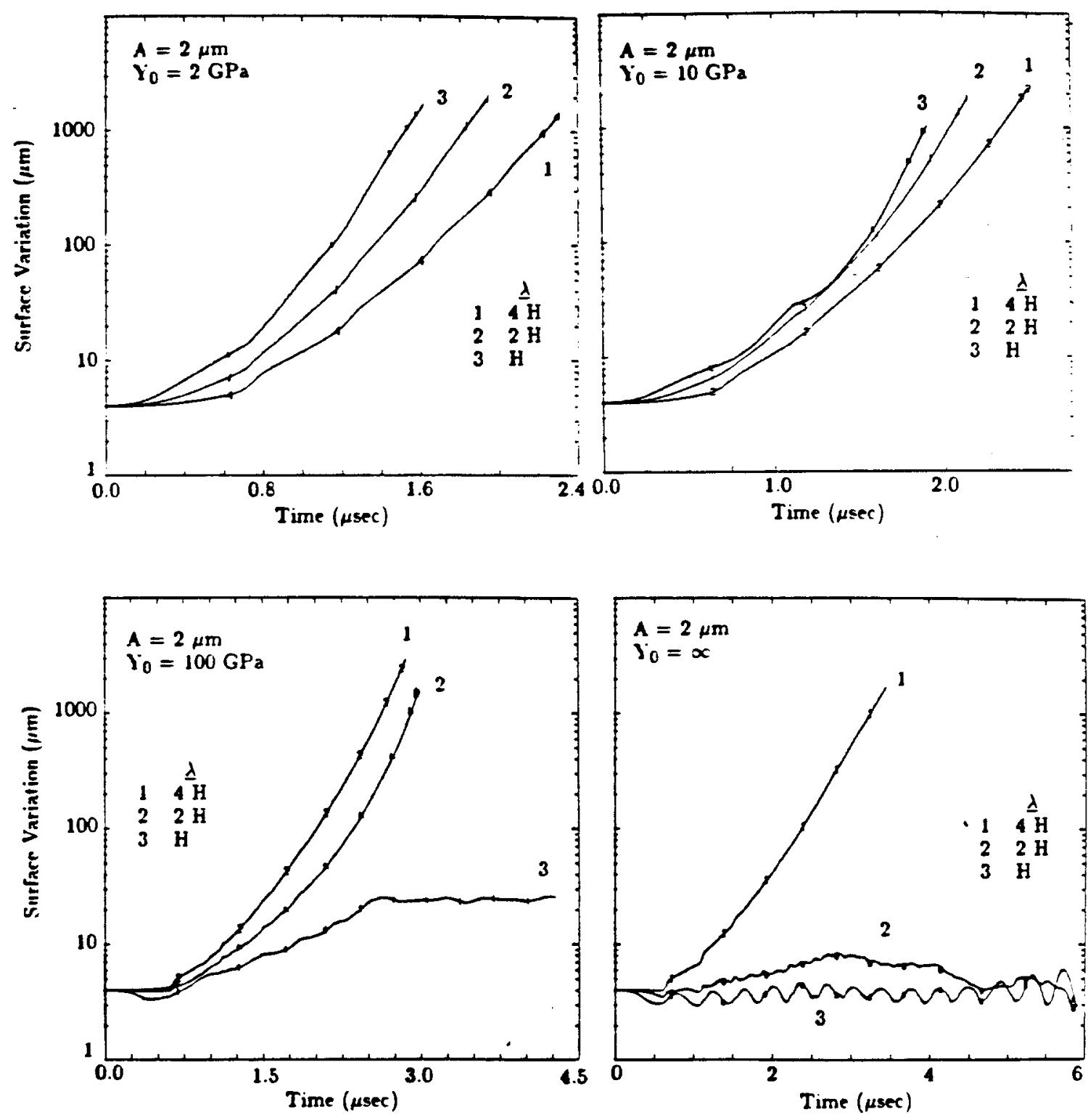

Figure 8: Variations in wavelength. 

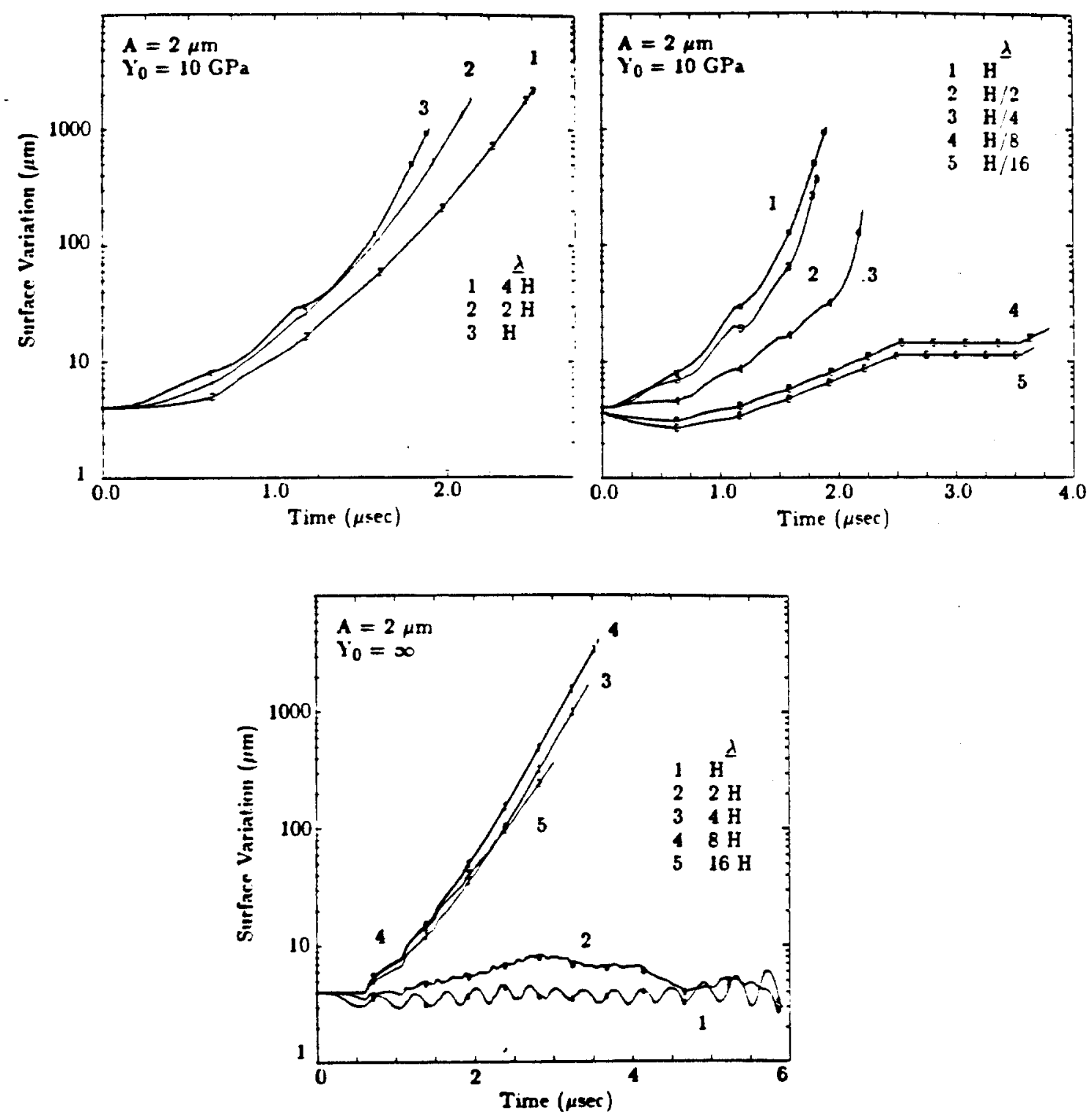

Figure 9: Demonstration of wavelength of maximum growth. 


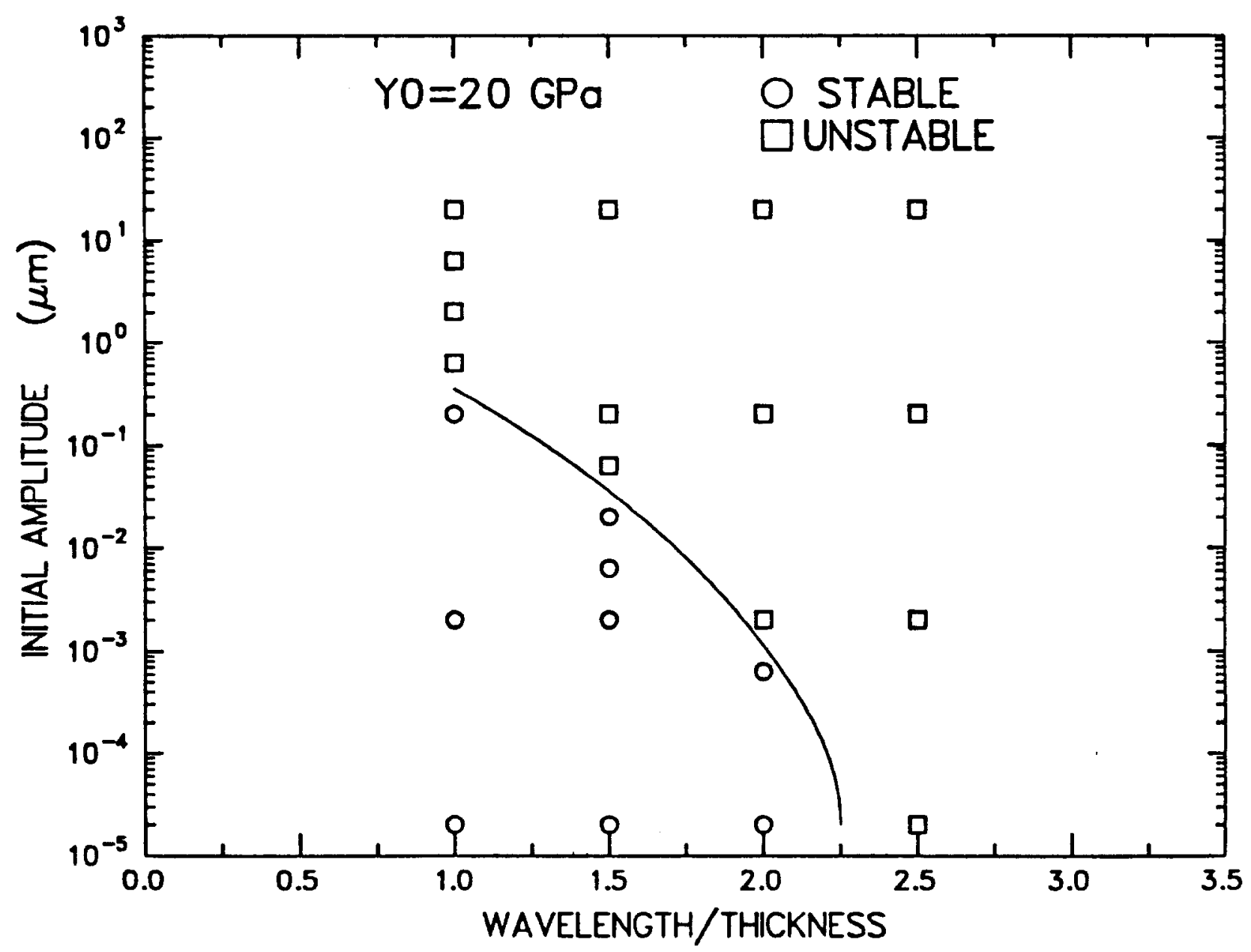

Figure 10: Amplitude-wavelength effect on stability. 

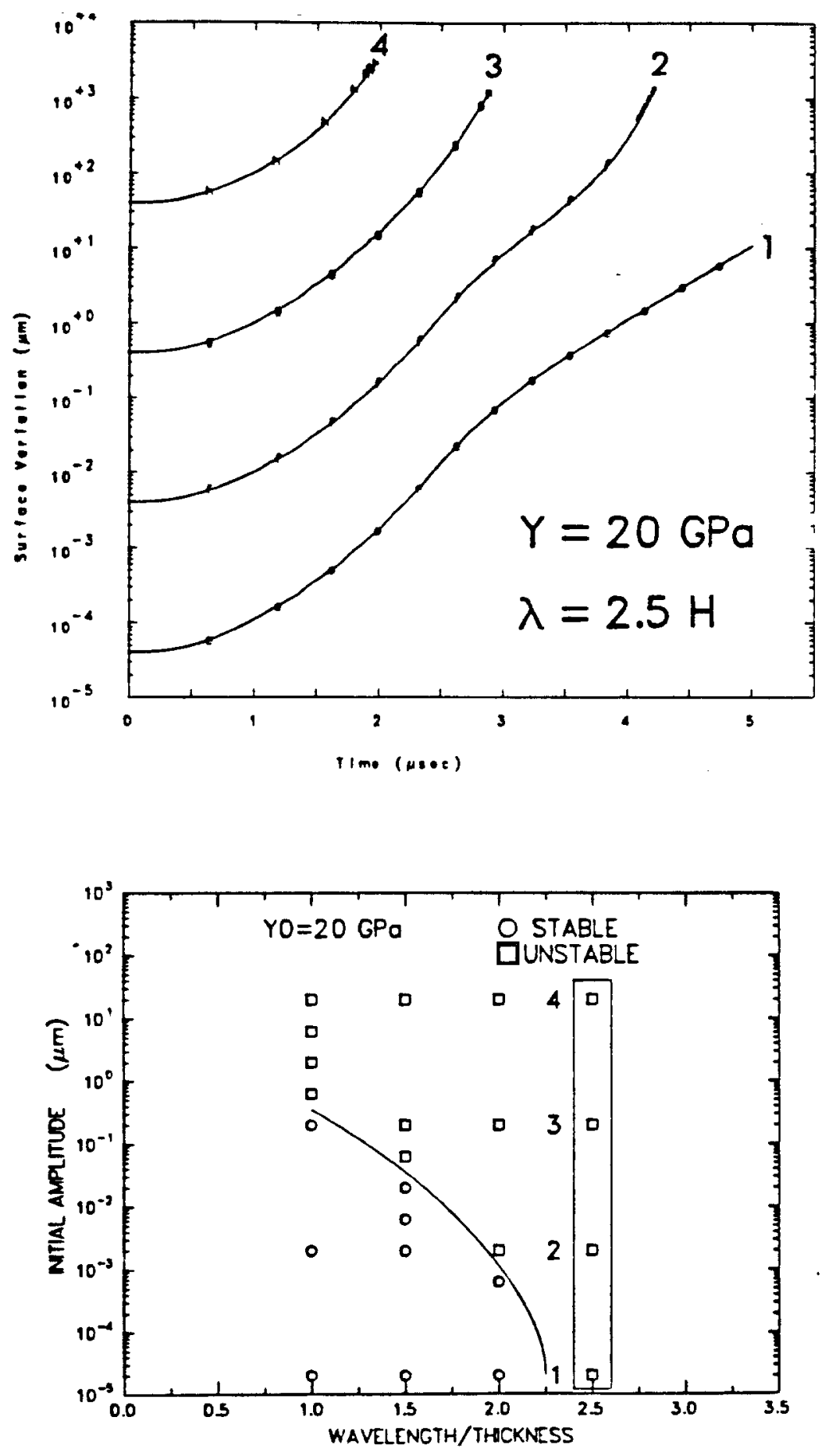

Figure 11: Response curves in unstable regime. 

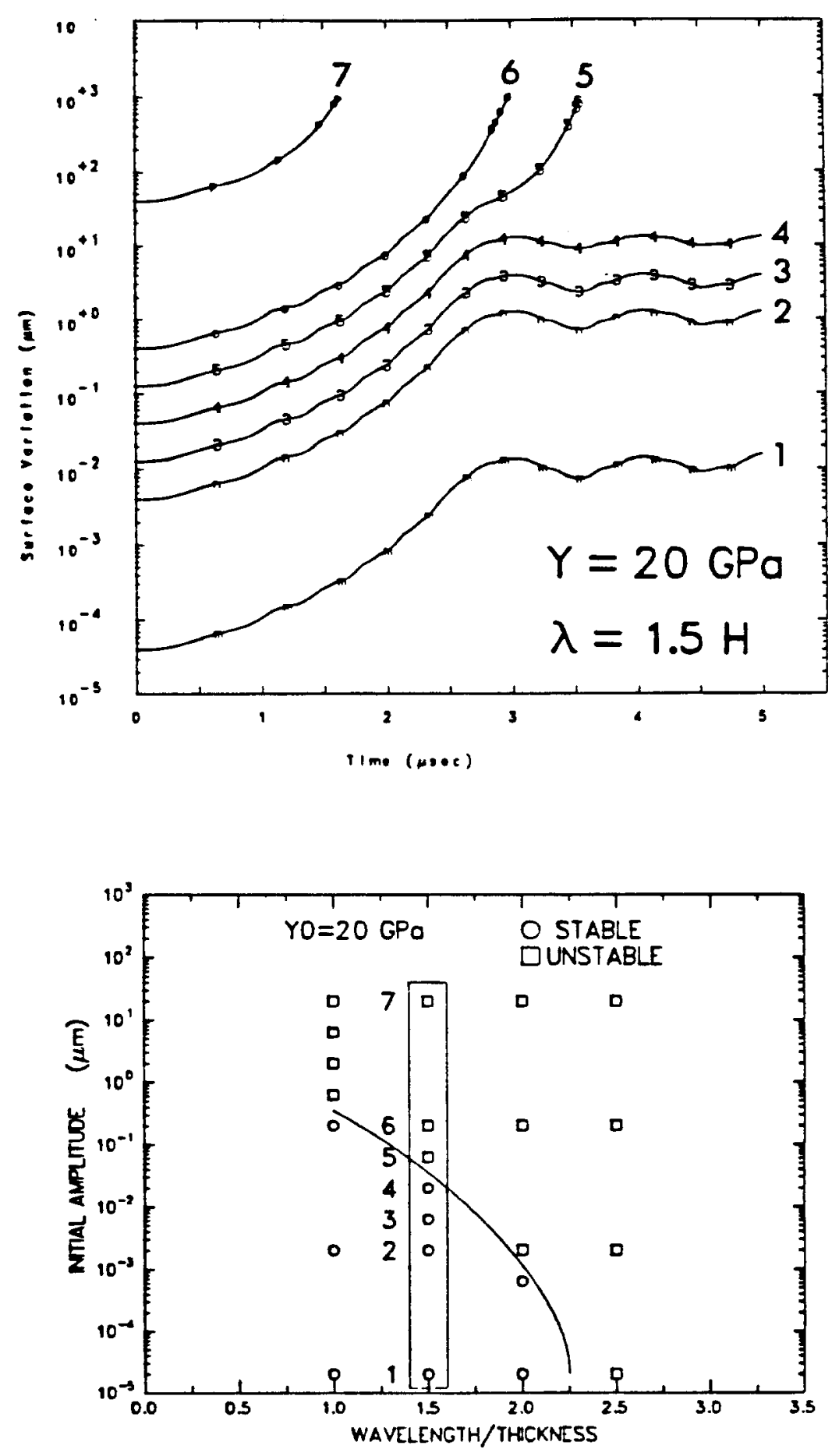

Figure 12: Response curves in amplitude dependent stability regime. 


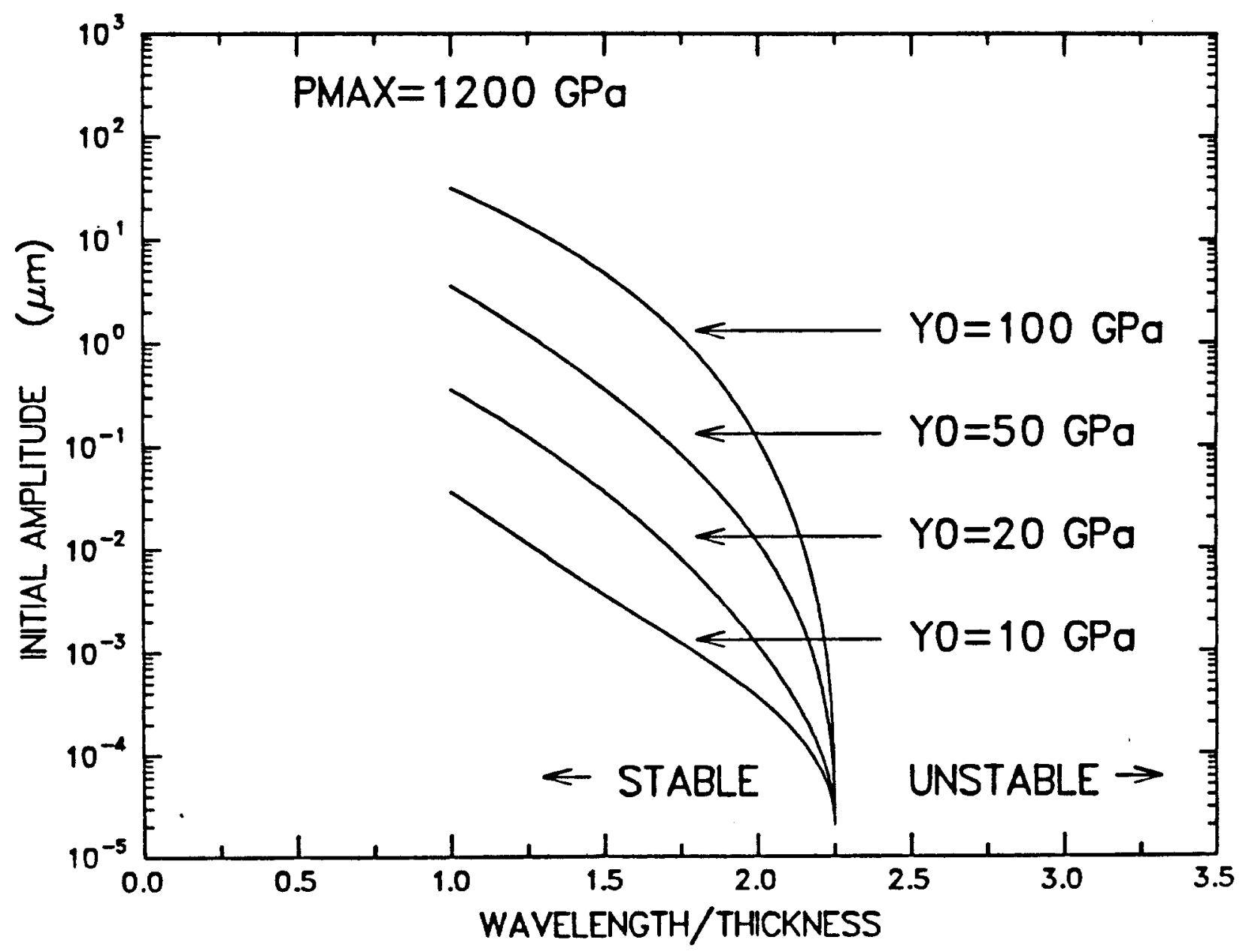

Figure 13: Yield strength effect on stability boundary. 


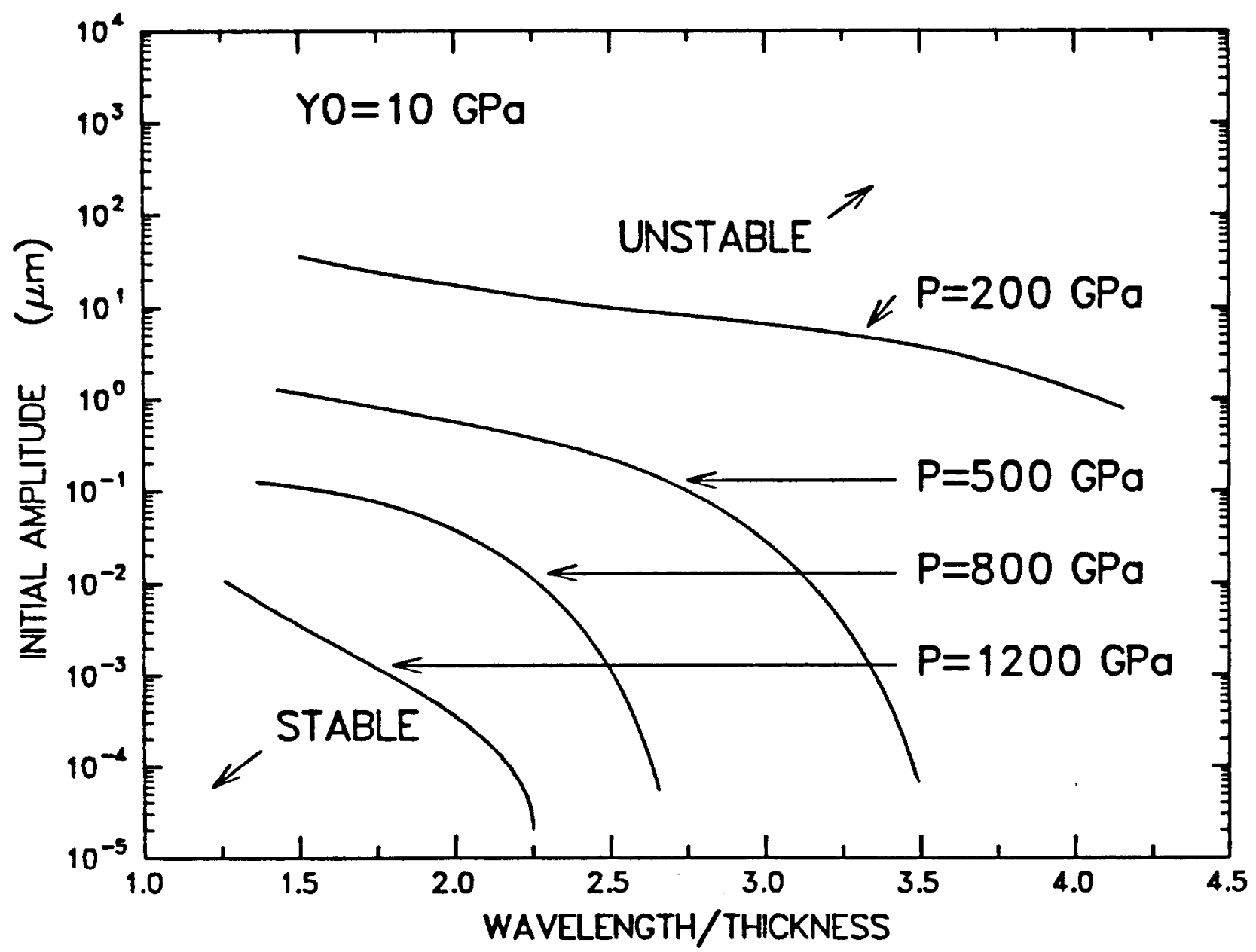

Figure 14: Driving pressure effect on stability boundary. 


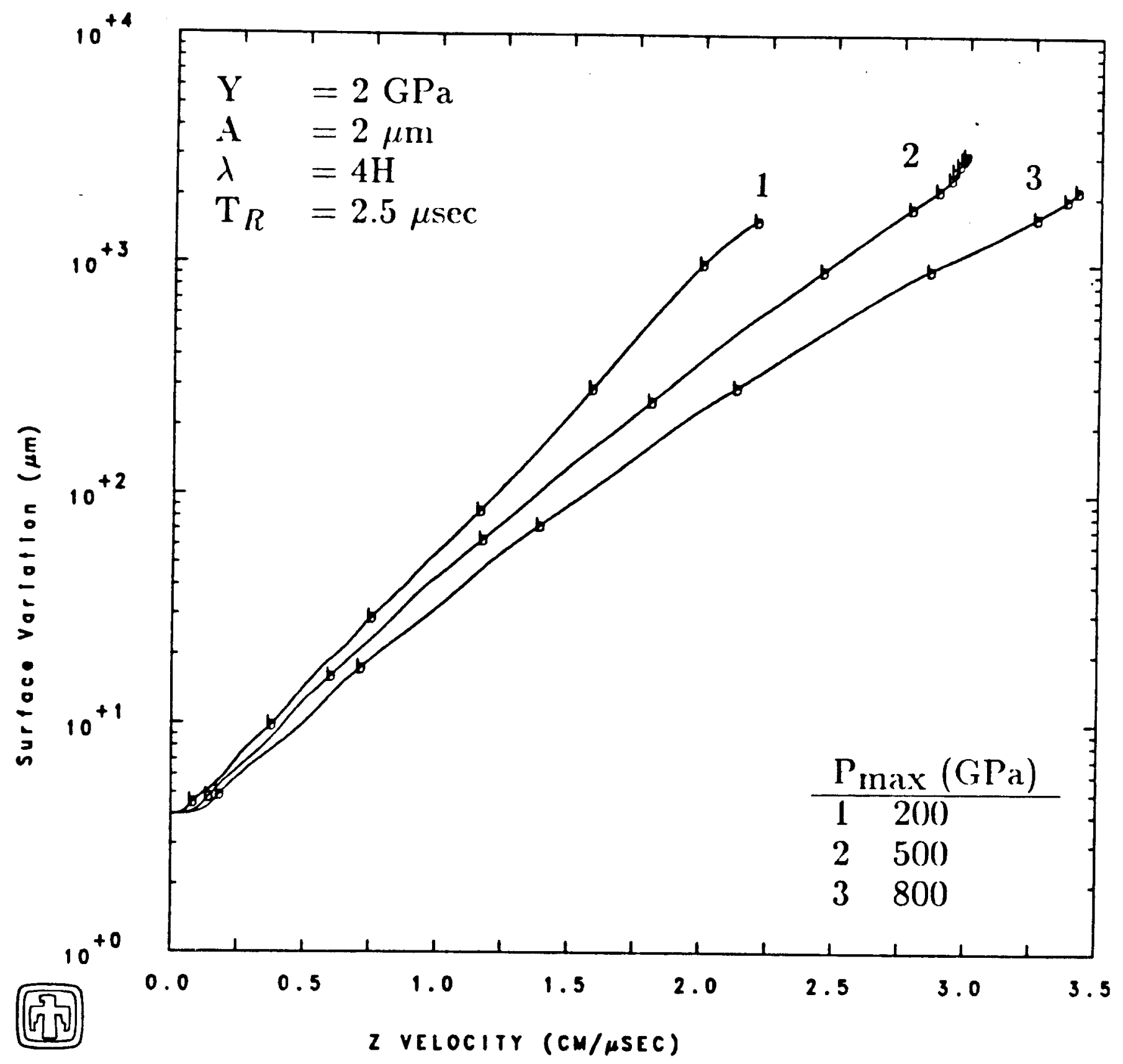

Figure 15: Instability growth vs. velocity. 

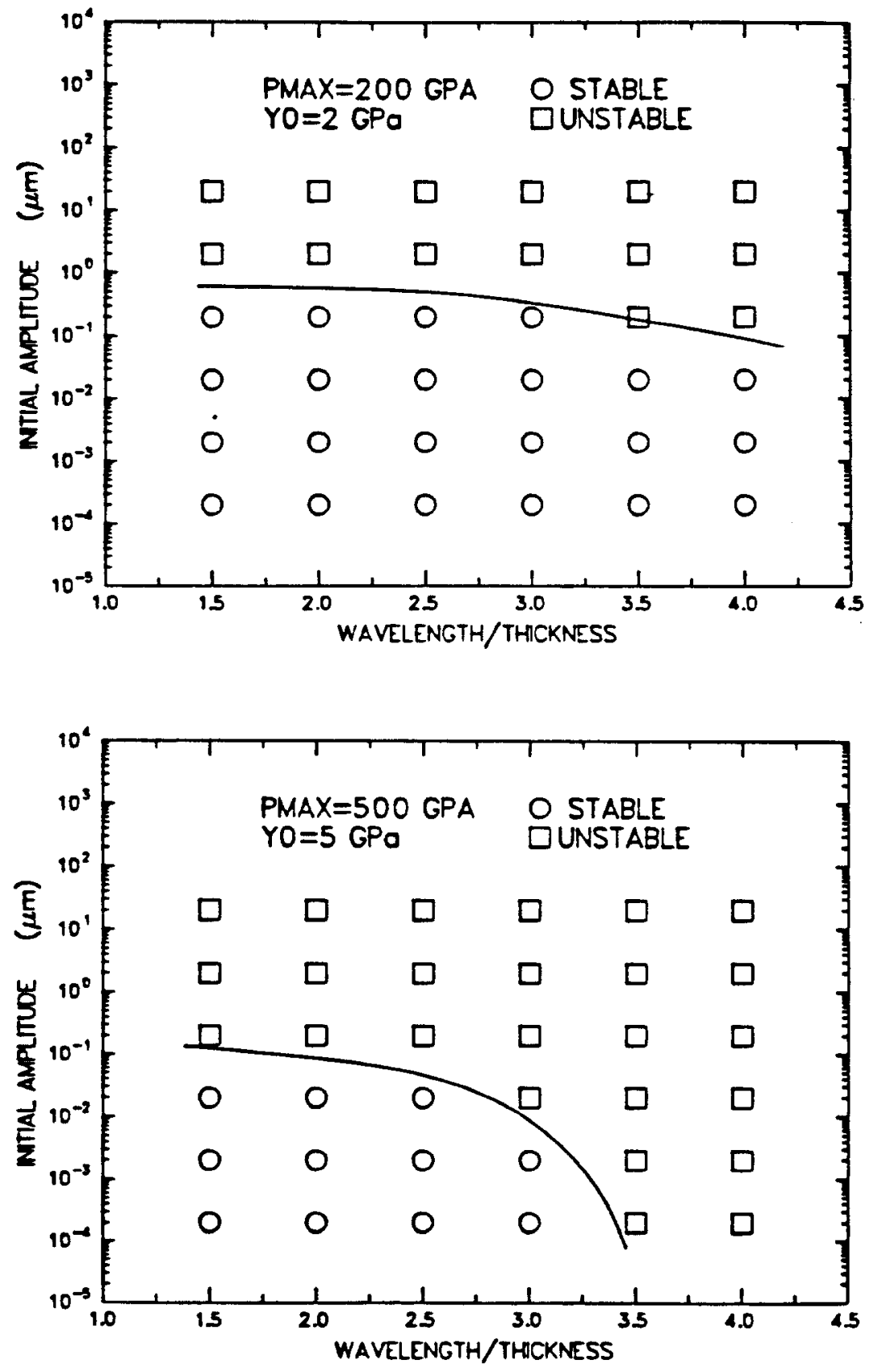

Figure 16: Amplitude-wavelength effect on stability. 


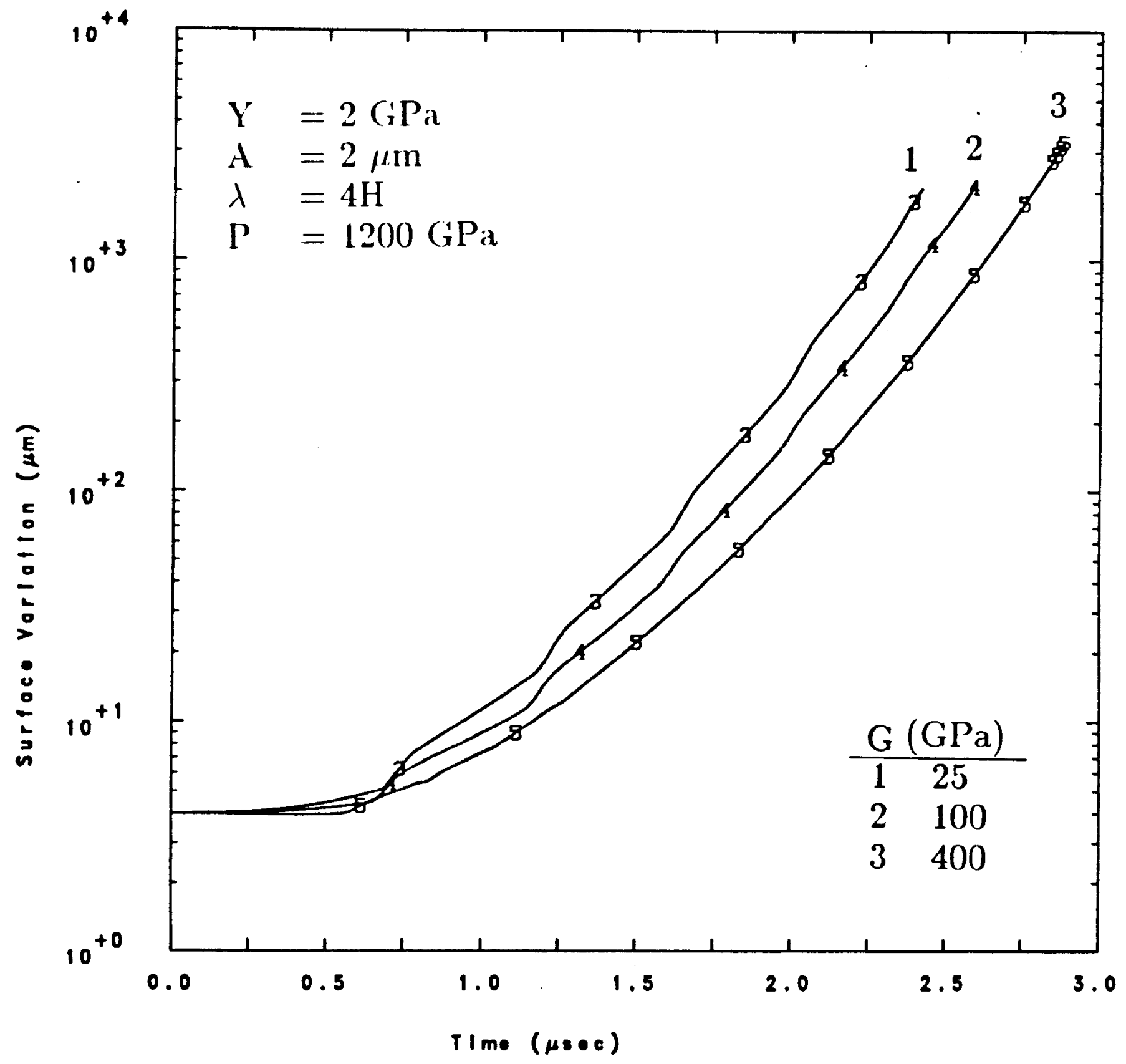

Figure 17: Shear modulus variations. 


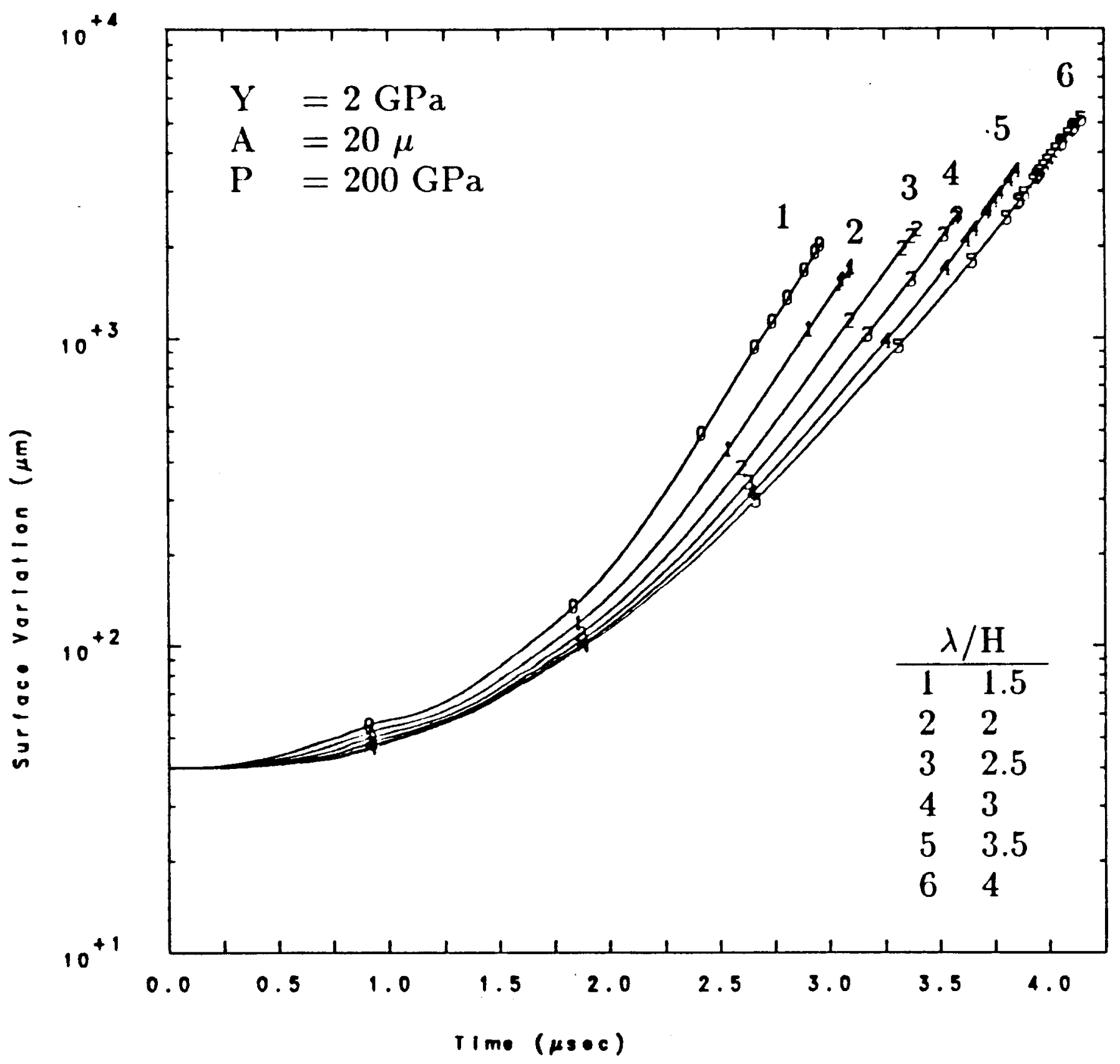

Figure 18: Wavelength variations in unstable regime. 


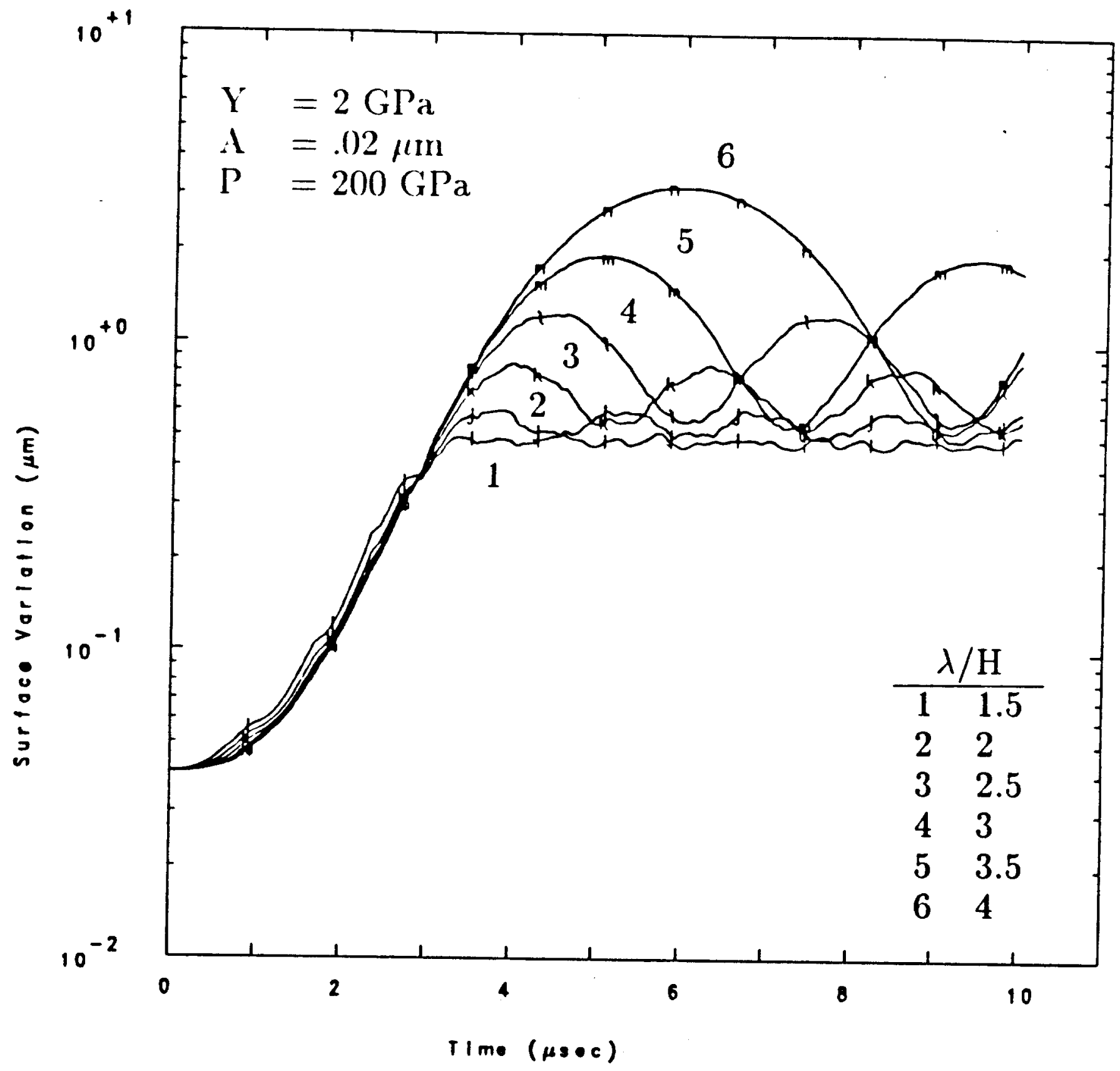

Figure 19: Wavelength variations in stable regime. 
PULSE RISETIME EFFECT ON STABILITY BOUNDARY

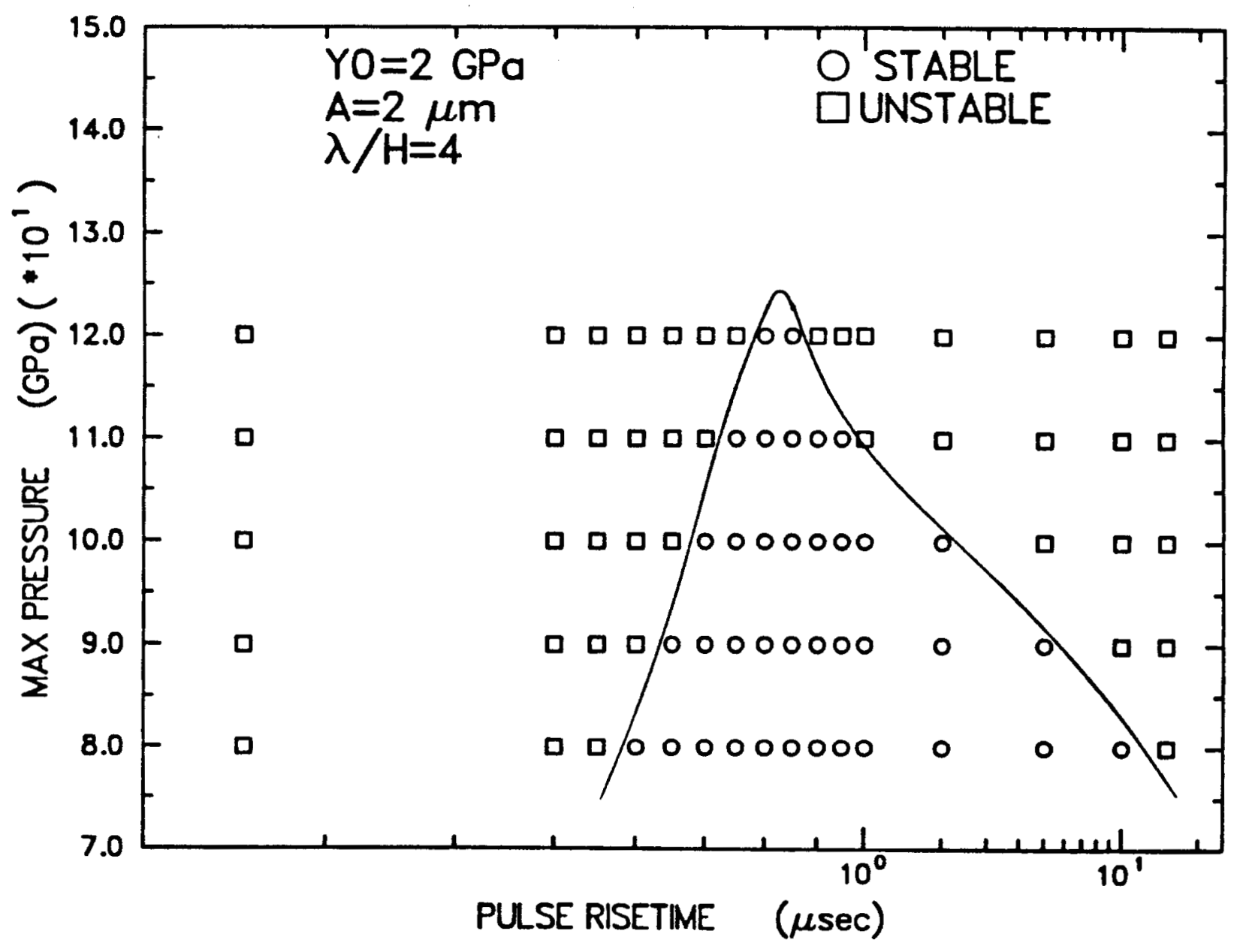

Figure 20: Pulse risetime effect on stability boundary. 


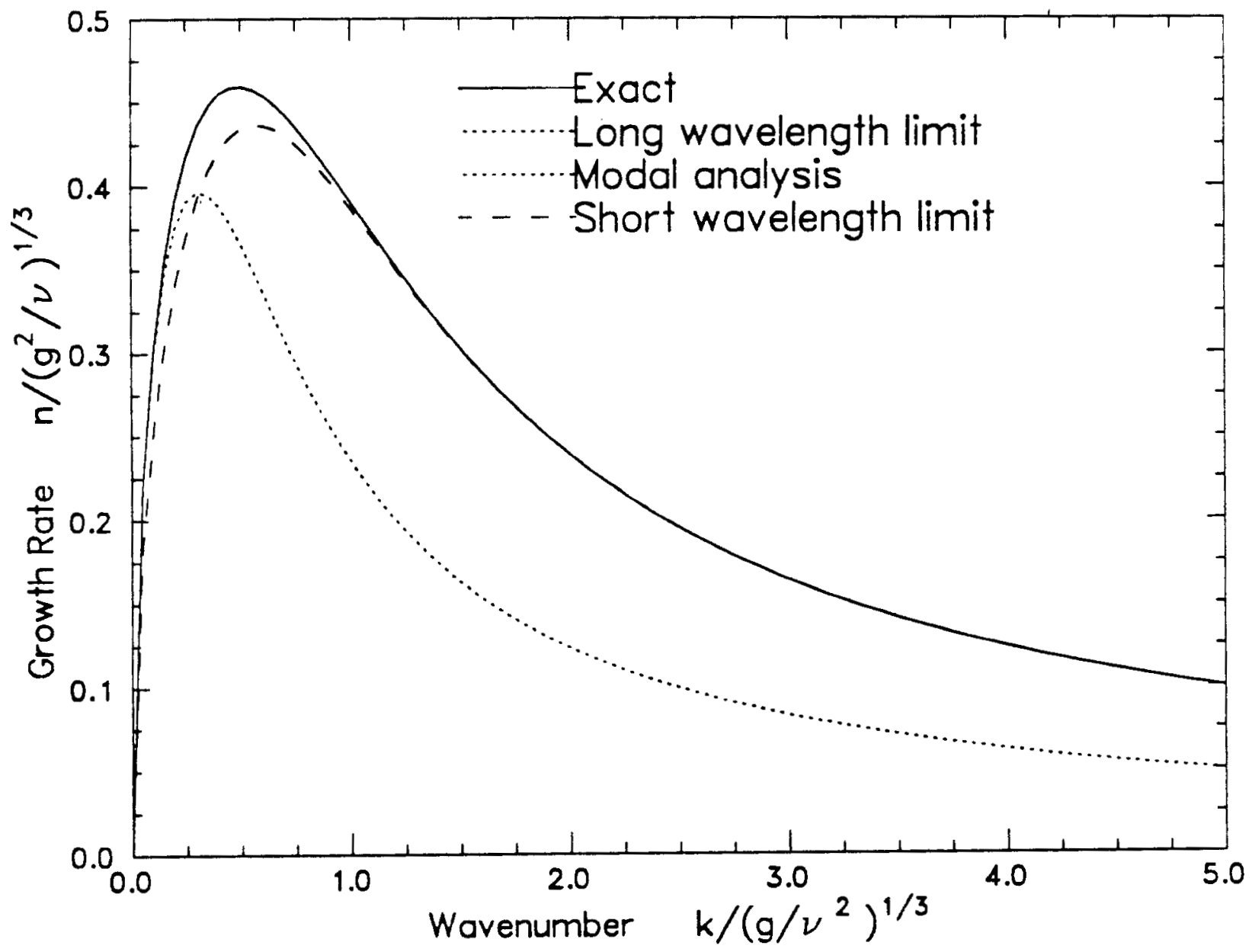

Figure 21: Newtonian fluid growth rates for $\rho_{2}=0$. 


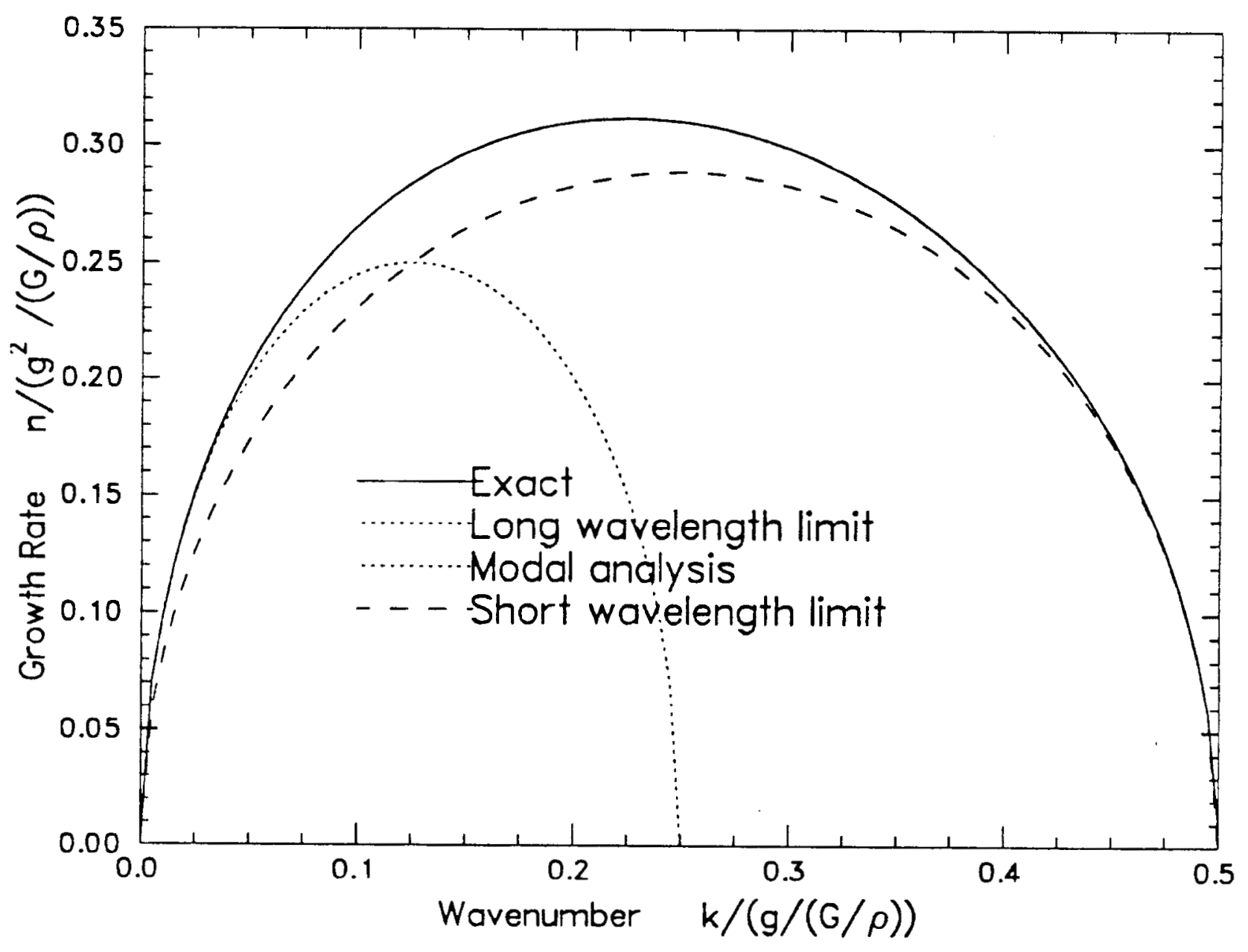

Figure 22: Elastic solid growth rates for $\rho_{2}=0$. 


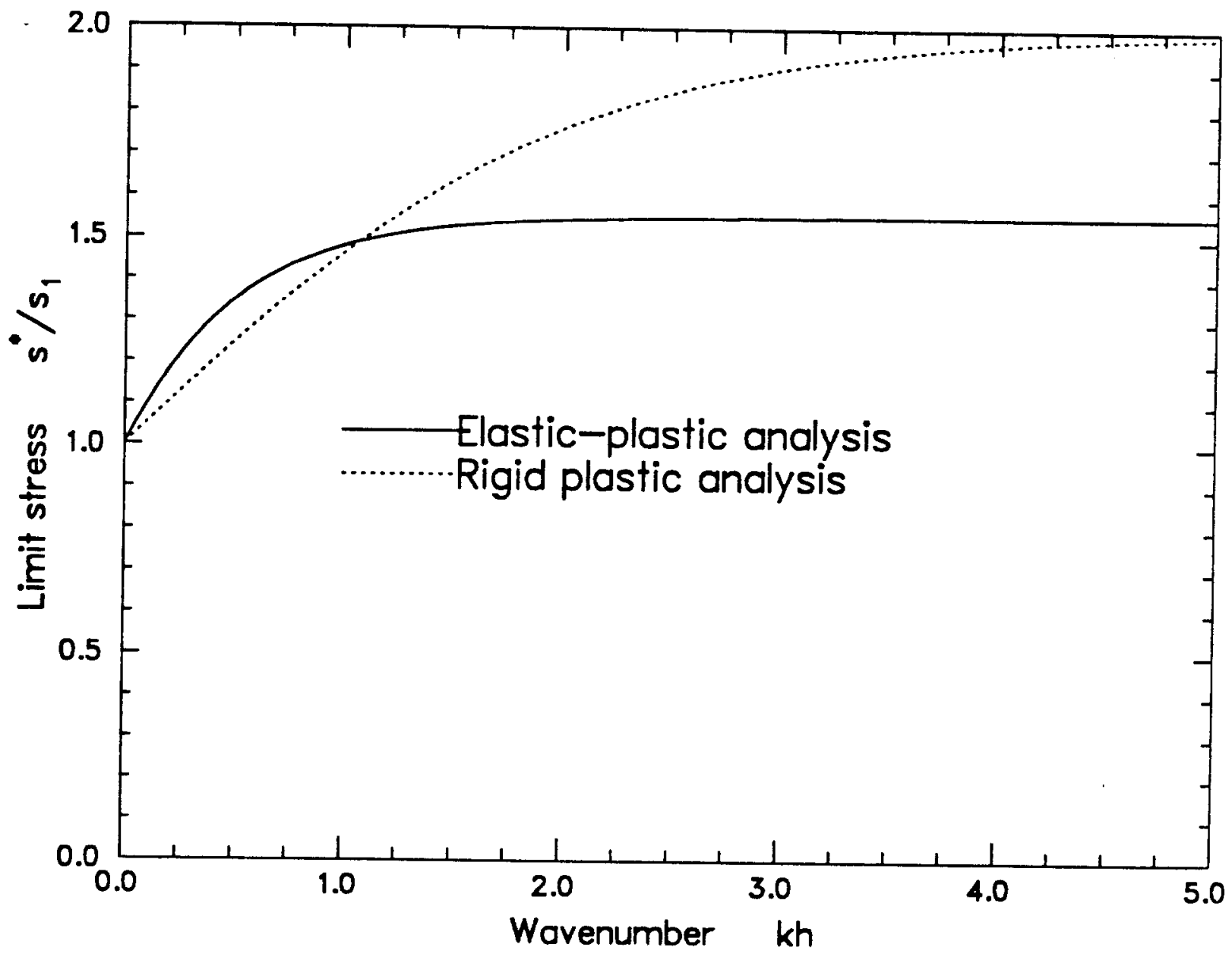

Figure 23: Comparison of limit stress versus wavenumber. 


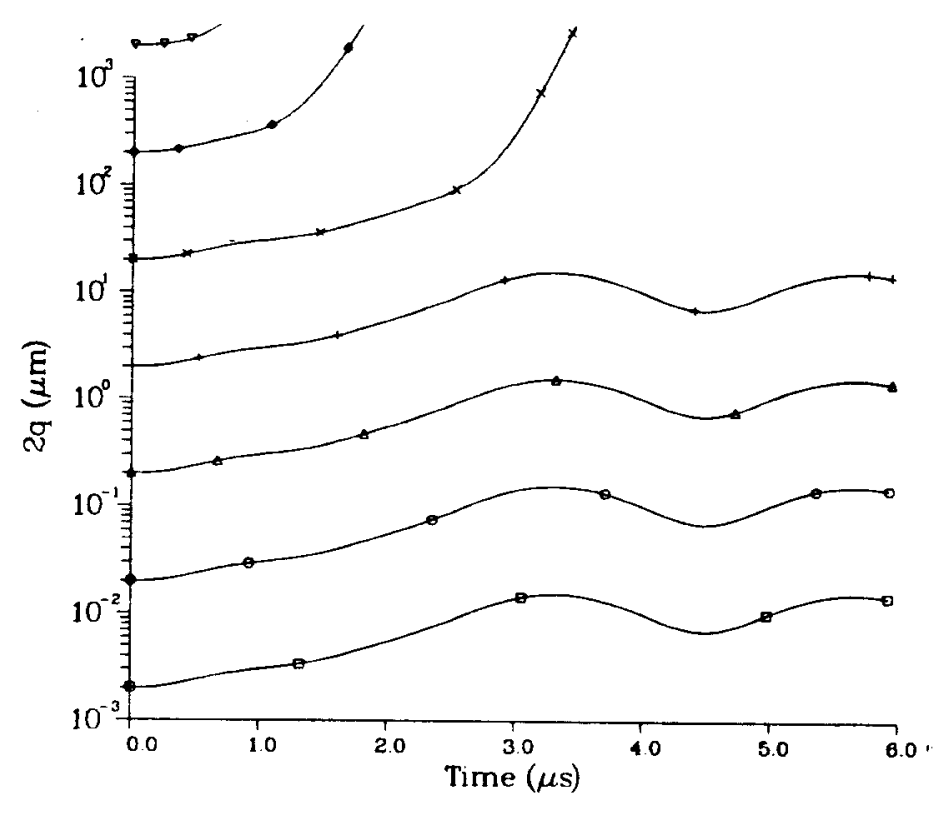

(a)

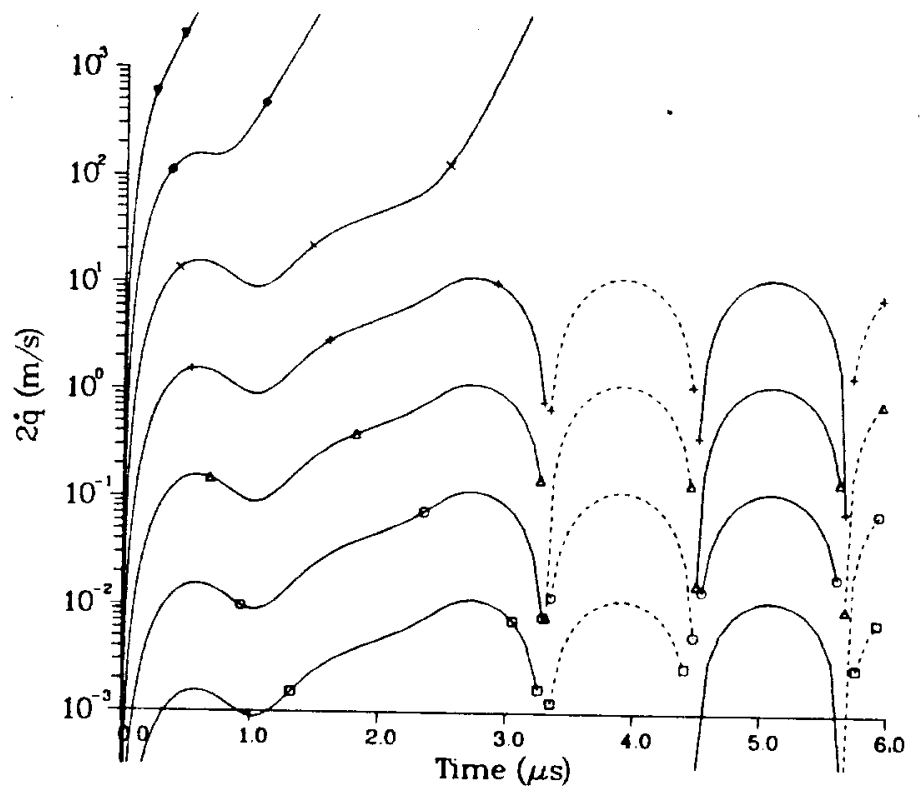

(b)

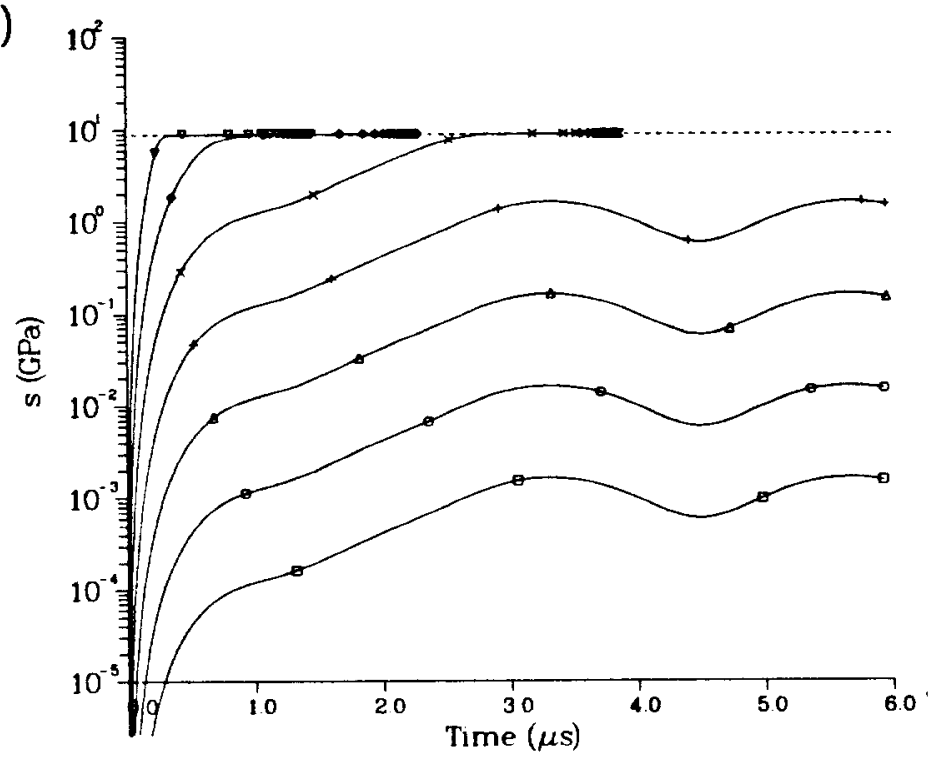

(c)

Figure 24: Solution to model equations. $\left(\lambda / h=2.0 ; p_{m}=800 \mathrm{GPa}\right)$ 


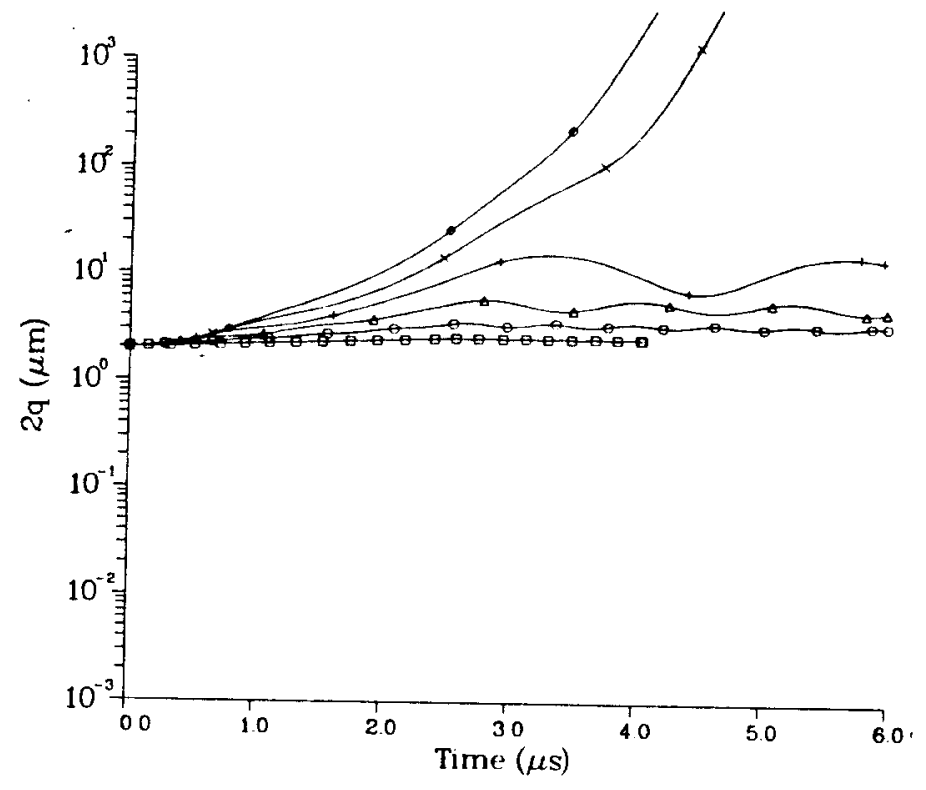

(a)

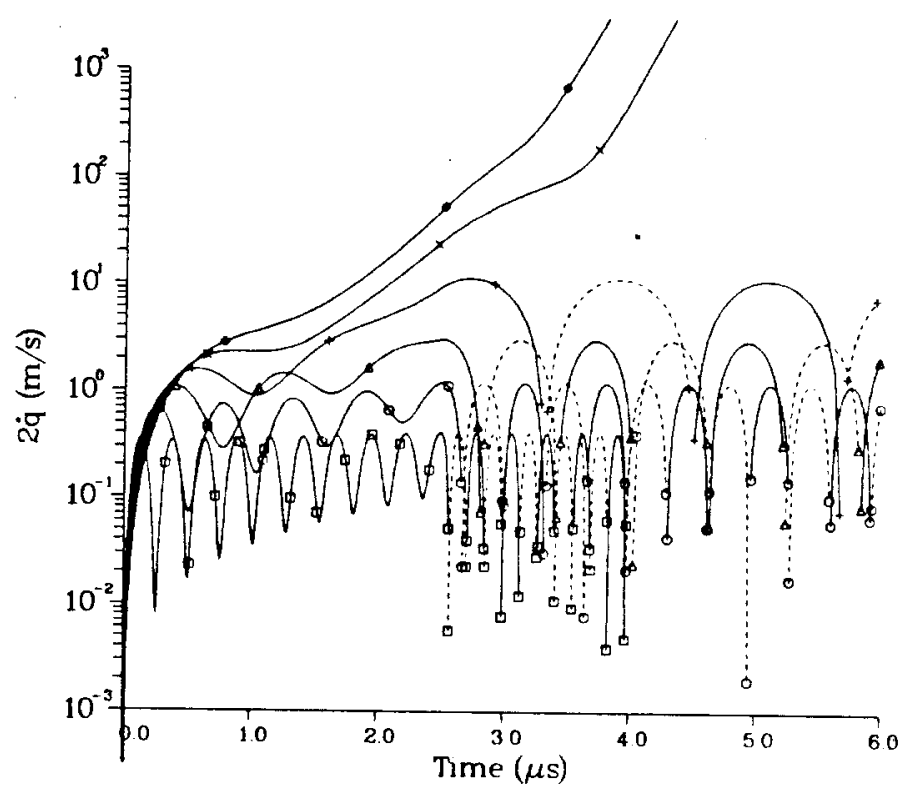

(b)

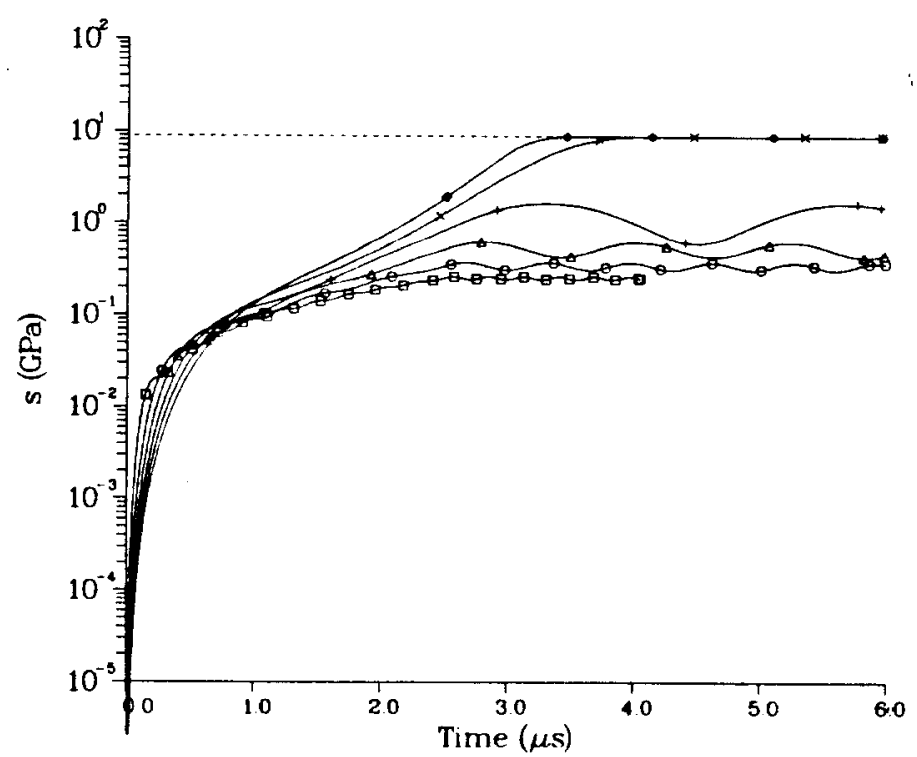

(c)

Figure 25: Solution to model equations. $\left(\lambda / h=.5,1.0,1.5,2.0,2.5,3.0 ; \mathrm{p}_{m}=800 \mathrm{GPa}\right)$ 


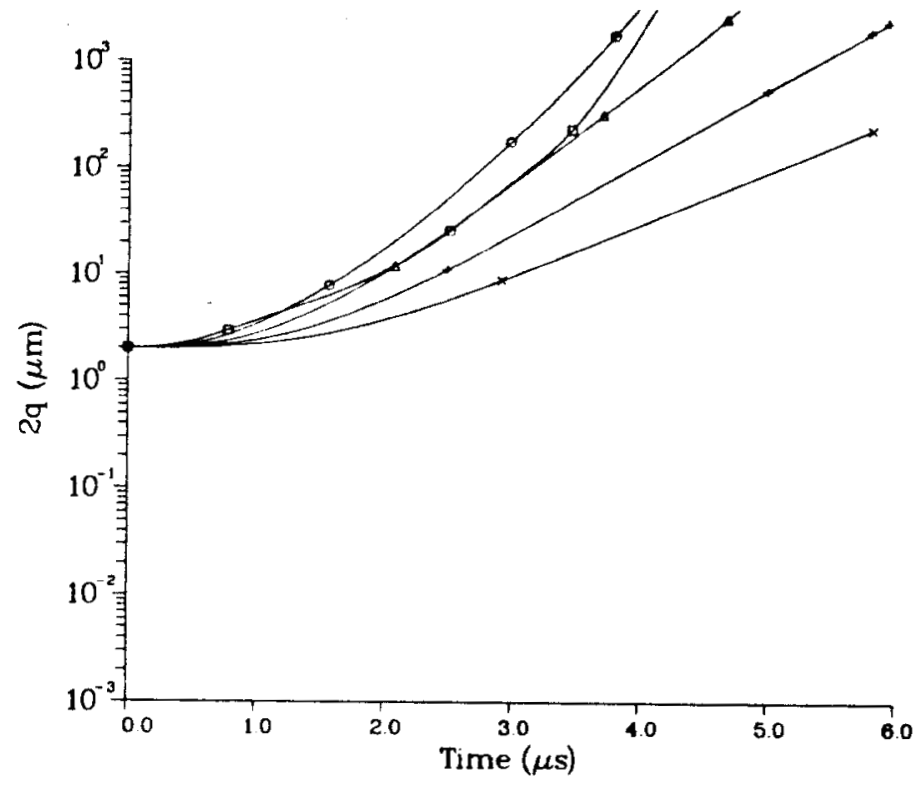

(a)

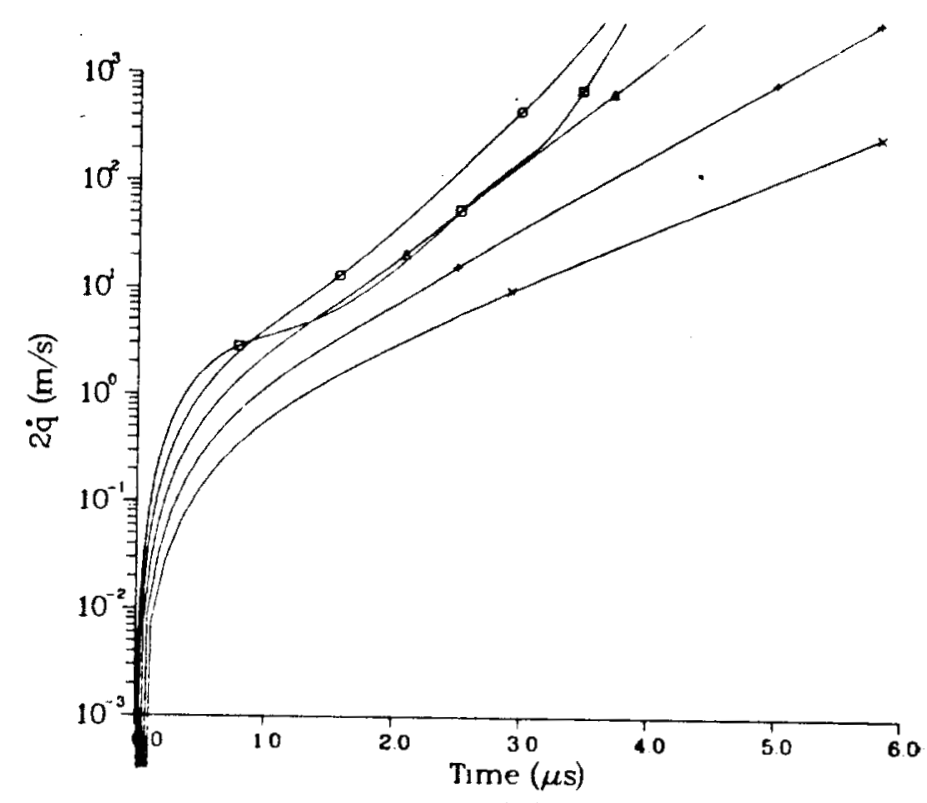

(b)

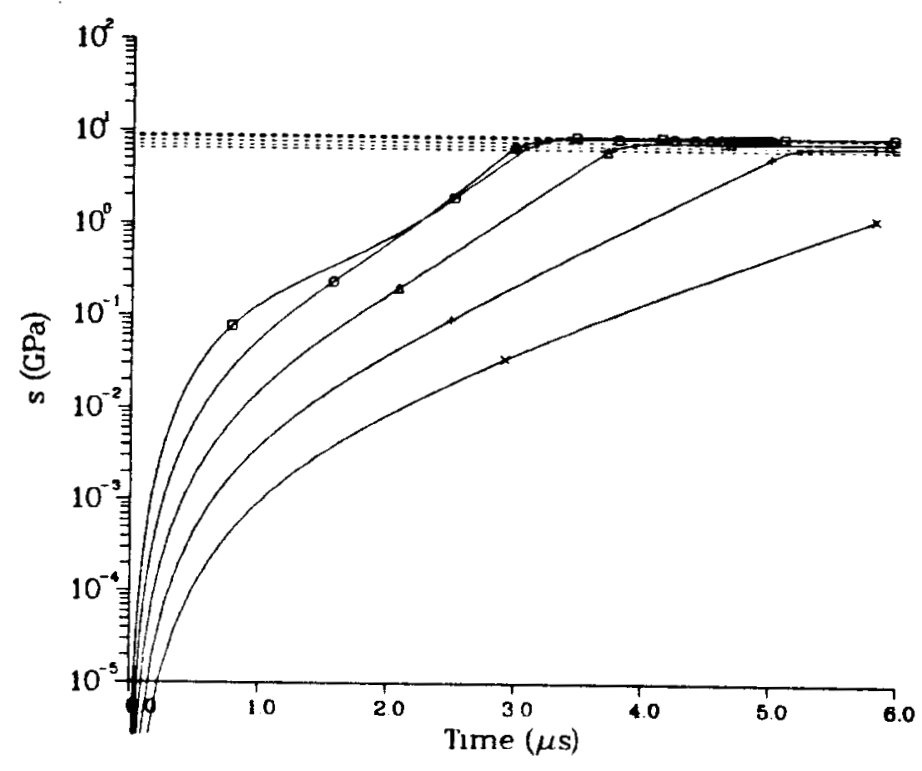

(c)

Figure 26: Solution to model equations. $\left(\lambda / h=3,6,12,24,48 ; \mathrm{p}_{m}=800 \mathrm{GPa}\right.$ ) 


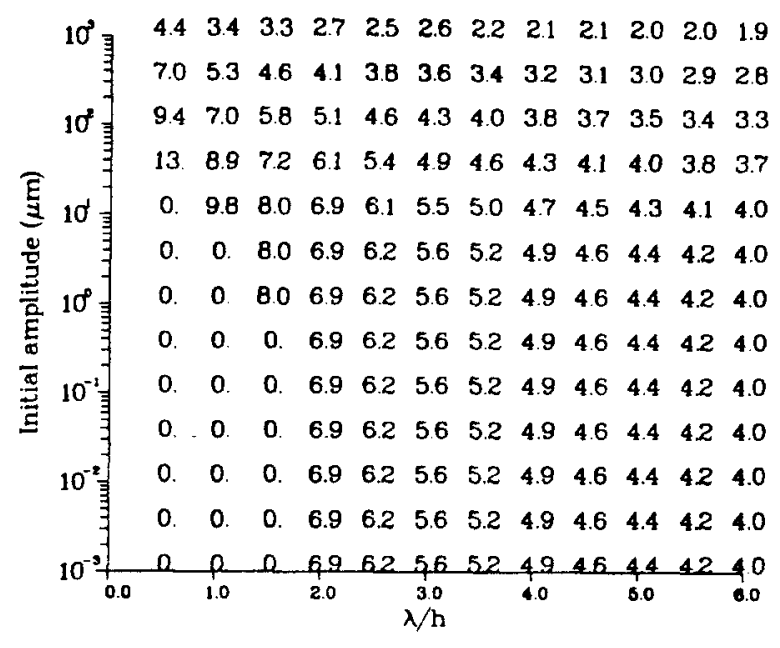

(a) $\mathrm{p}_{m}=1200 \mathrm{GPa}$

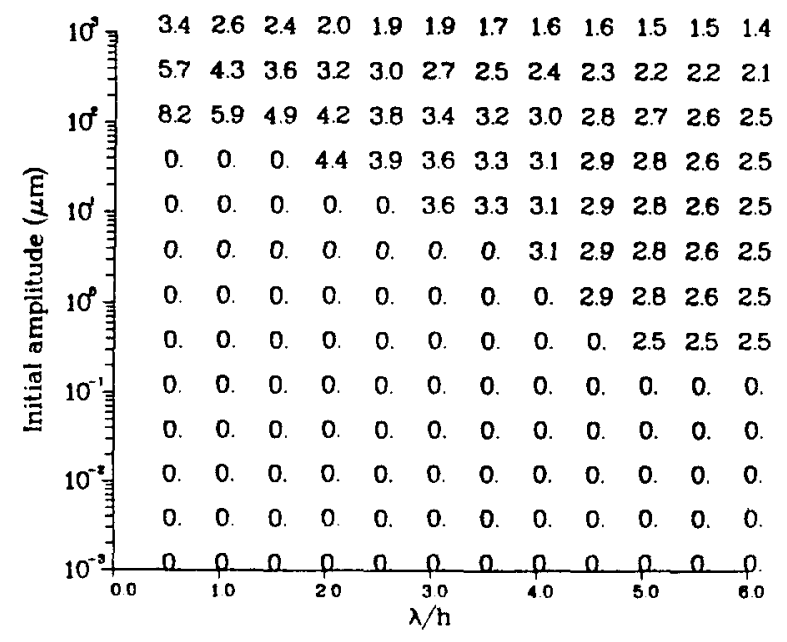

(c) $\mathrm{p}_{m}=500 \mathrm{GPa}$

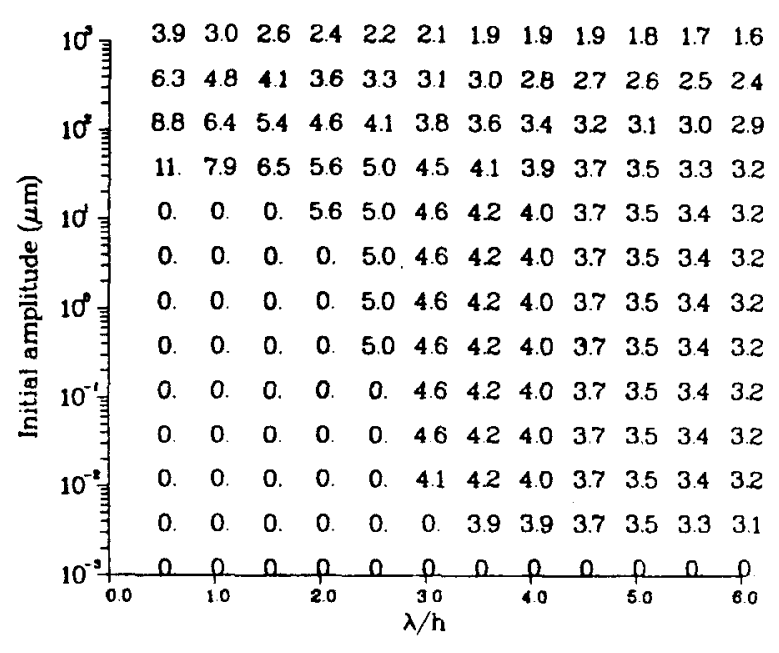

(b) $\mathrm{p}_{m}=800 \mathrm{GPa}$

(d) $\mathrm{p}_{m}=200 \mathrm{GPa}$

Figure 27: A comparison of final growth rates in $(\mu s)^{-1}$. 


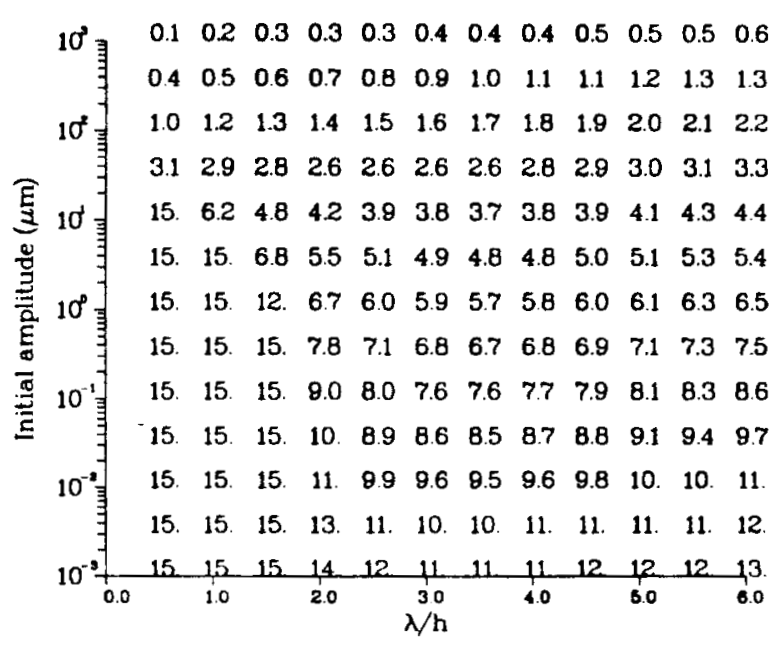

(a) $\mathrm{p}_{m}=1200 \mathrm{GPa}$

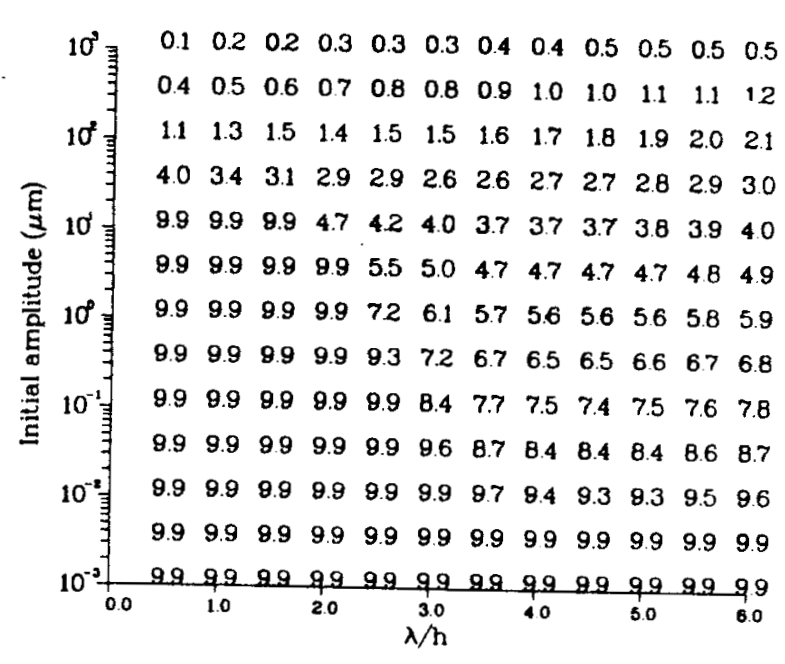

(b) $\mathrm{p}_{m}=800 \mathrm{GPa}$

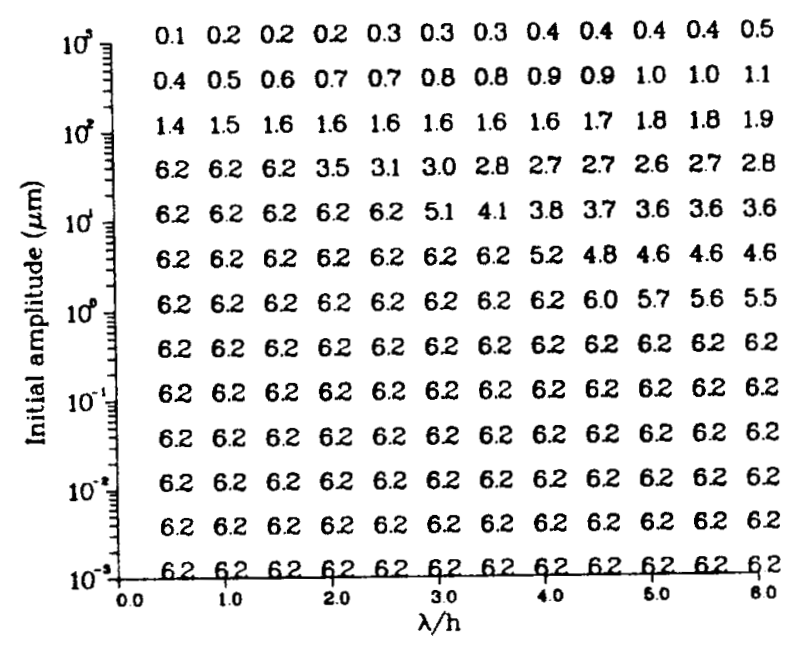

(c) $\mathrm{p}_{m}=500 \mathrm{GPa}$

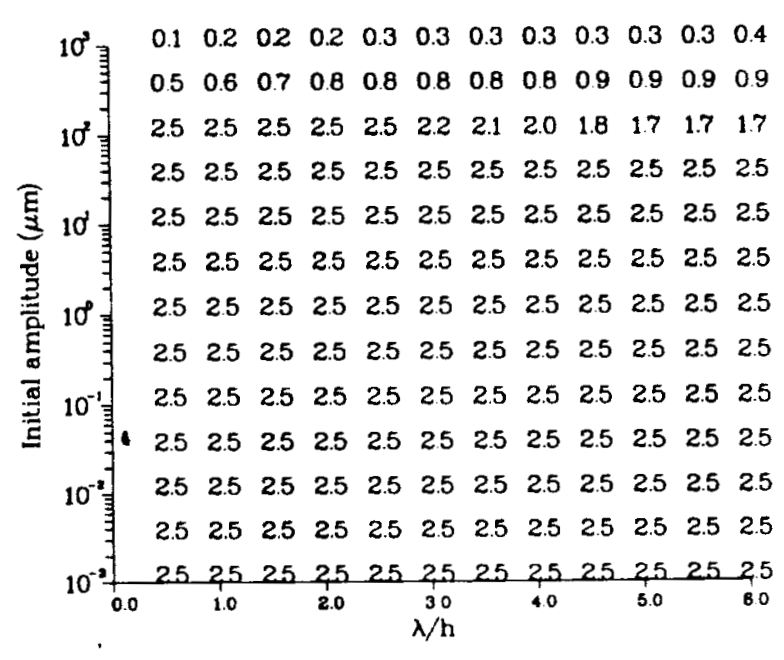

(d) $\mathrm{p}_{m}=200 \mathrm{GPa}$

Figure 28: A comparison of plate velocity in $\mathrm{cm} / \mu \mathrm{s}$. 


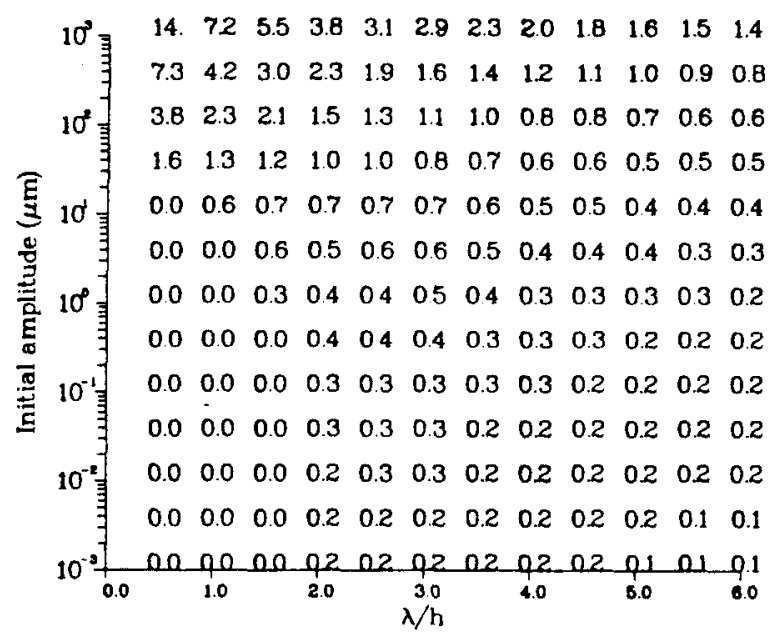

(a) $\mathrm{p}_{m}=1200 \mathrm{GPa}$

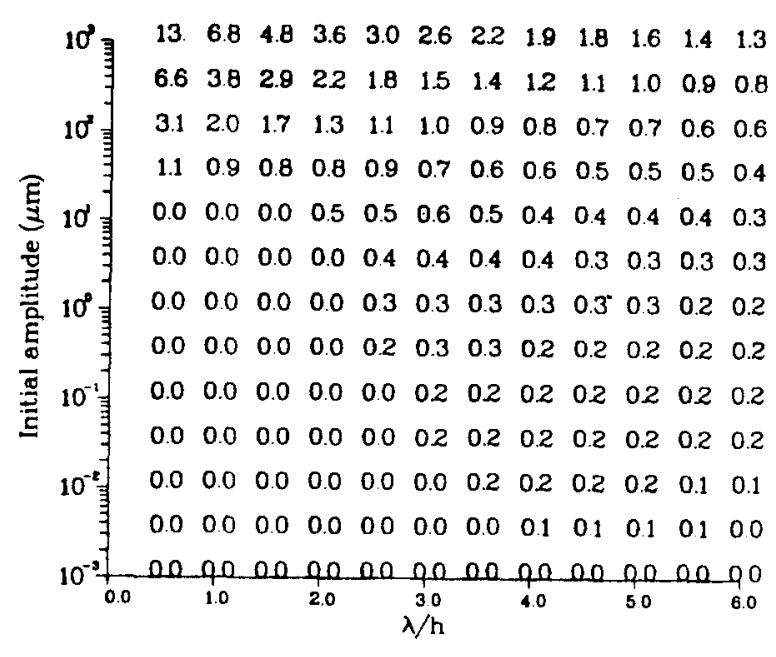

(b) $\mathrm{p}_{m}=800 \mathrm{GPa}$ (c) $\mathrm{p}_{m}=500 \mathrm{GPa}$

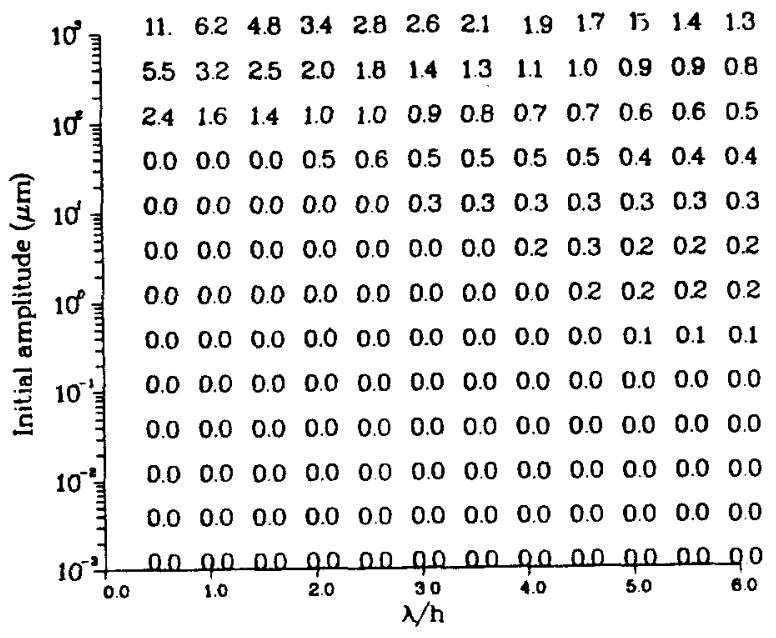

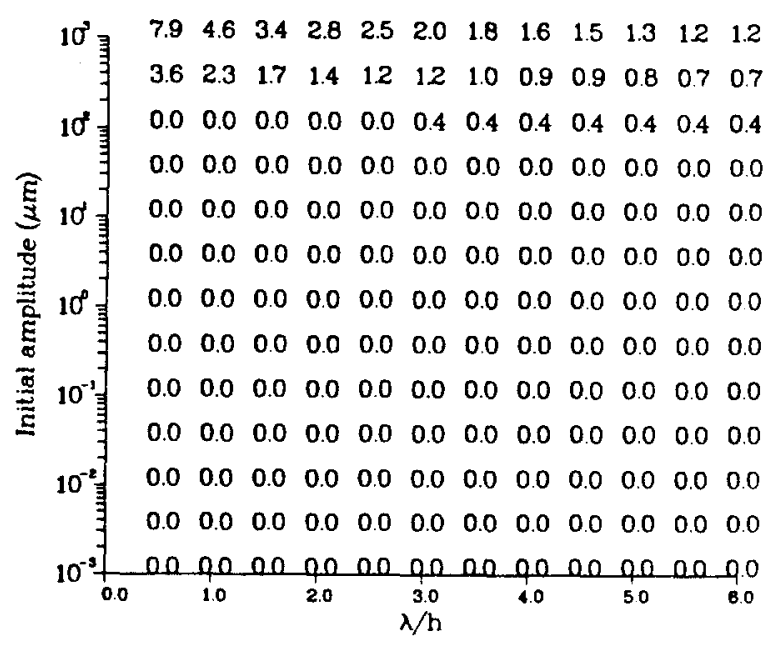

(d) $\mathrm{p}_{m}=200 \mathrm{GPa}$

Figure 29: A comparison of relative velocity deviation $\delta v / v \equiv 2 \dot{q} / v$. 


\section{References}

1. J. F. Barnes, P. J. Blewett, R. G. McQueen, K. A. Meyer, and D. Venable, "Taylor Instability in Solids," J. Appl. Phys., 45 (2), 1974, pp. 727-732.

2. J. F. Barnes, D. H. Janney, R. K. London, K. A. Meyer and D. H. Sharp, "Further Experimentation on Taylor Instability in Solids," J. Appl. Physics, 51 (9), 1980, pp. 4678-4679.

3. S. Chandrasekhar, "Hydrodynamic and Hydromagnetic Stability," Dover Publications, Inc., New York, 1981, pp. 441-443.

4. J. K. Dienes, "Method of generalized coordinates and an application to RayleighTaylor Instability," Phys. Fluids 21(5), May 1978, pp. 736-744.

5. D. C. Drucker, "Taylor Instability of the Surface of an Elastic-Plastic Plate," Mechanics Today, Vol. 5, S. Nemmat-Nasser, Ed., 1980, pp. 37-47.

6. D. C. Drucker, "A Further Look at Rayleigh-Taylor and Other Surface Instabilities in Solids," Ingenieur-Archiv 49, (1980), pp. 361-367.

7. J. W. Miles, "Taylor Instability of a Flat Plate," General Dynamics GAMD-7335, AD643161, 1966.

8. J. W. Miles and J. K. Dienes, "Taylor Instability in a Viscous Liquid," Phys. of Fluids, 9 (12), 1966, pp. 2518-2519.

9. D. H. Sharp, “An Overview of Rayleigh-Taylor Instability," Physica 12D, 1984, pp. 3-18.

10. J. W. Swegle, "TOODY IV - A Computer Program for Two-Dimensional Wave Propagation", SAND78-0552, 1978.

11. G. N. White, "A One-Degree-of-Freedom Model for the Taylor Instability of an Ideally Plastic Metal Plate," Los Alamos National Laboratory, LA-5225-MS, Informal Report, April 1973. 


\section{DISTRIBUTION:}

J. F. Barnes, T-1, MS B221

Los Alamos National Laboratory

P. O. Box 1663

Los Alamos, NM 87545

P. Blewett, X-5, MS F669

Los Alamos National Laboratory

P. O. Box 1663

Los Alamos, NM 87545

B. Daly, T-3, MS B216

Los Alamos National Laboratory

P. O. Box 1663

Los Alamos, NM 87545

J. Dienes, T-3, MS B216

Los Alamos National Laboratory

P. O. Box 1663

Los Alamos, NM 87545

S. W. Eisenhawer, Q-6, MS K557

Los Alamos National Laboratory

P. O. box 1663

Los Alamos, NM 87545

R. P. Godwin, X-3, MS F669

Los Alamos National Laboratory

P. O. Box 1663

Los Alamos, NM 87545

F. Harlow, T-3, MS B216

Los Alamos National Laboratory

P. O. Box 1663

Los Alamos, NM 87545

N. Johnson, T-3, MS B216

Los Alamos National Laboratory

P. O. Box 1663

Los Alamos, NM 87545

T. A. Sandford, DRA/NDR, MS F617

Los Alamos National Laboratory

P. O. Box 1663

Los Alamos, NM 87545
G. R. Spillman, DRA/NDR, MS F617

Los Alamos National Laboratory

P. O. Box 1663

Los Alamos, NM 87545

J. W. Taylor, ADDRA, MS A110

Los Alamos National Laboratory

P. O. Box 1663

Los Alamos, NM 87545

Dr. Moe Sharff

Science Applications, Inc.

10401 Rosella Street

San Diego, CA 92121

Dr. Yasuyuki Horie

North Carolina State University

Box 7908

Rayleigh, NC 27695

\section{Sandia Internal}

1200 J. P. VanDevender

1271 T. W. Hussey

1500 W. Herrmann

1510 J. W. Nunziato

1513 M. R. Baer

1520 C. W. Peterson

1530 L. W. Davison

1531 S. L. Thompson

1531 T. K. Bergstresser

1531 S. L. Passman

1531 J. S. Rottler

1531 J. W. Swegle (20)

1533 P. Yarrington

1533 P. J. Chen

1533 A. C. Robinson (20)

1534 J. R. Asay

1534 L. C. Chhabildas

1534 J. E. Dunn

1534 D. E. Grady

1534 T. G. Trucano

1550 R. C. Maydew

3141 S. A. Landenberger (5) 
3151 W. L. Garner (3)

3154-3 C. H. Dalin (28)

For DOE/TIC

$5100 \mathrm{H}$. W. Schmitt

5160 G. R. Otey

5164 J. F. Cuderman

5164 H. P. Fell

5164 S. W. Hatch

8024 P. M. Dean

8400 R. C. Wayne

8430 J. Vitko

$8434 \mathrm{~J}$. B. Woodard

8434 T. E. Owen

8434 C. J. Pignolet

8434 C. A. Pura

8470 R. L. Rinne

8478 M. E. John

9012 J. W. Keizur

9012 R. J. Lawrence

9012 R. M. Zazworsky 